\title{
THE SDSS-II SUPERNOVA SURVEY: PARAMETERIZING THE TYPE Ia SUPERNOVA RATE AS A FUNCTION OF HOST GALAXY PROPERTIES
}

\author{
Mathew Smith ${ }^{1,2,3}$, Robert C. Nichol ${ }^{3}$, Benjamin Dilday $^{4,5}$, John Marriner ${ }^{6}$, Richard Kessler $^{7,8}$, Bruce Bassett $^{9,10,11}$, \\ David Cinabro $^{12}$, Joshua Frieman ${ }^{6,7,8}$, Peter Garnavich ${ }^{13}$, Saurabh W. Jha $^{14}$, Hubert Lampeitl ${ }^{2}$, Masao Sako ${ }^{15}$, \\ DONALD P. SCHNEIDER ${ }^{16,17}$, AND JeSPER SOLlERMAN ${ }^{18}$ \\ ${ }^{1}$ Department of Physics, University of Western Cape, Bellville 7530, Cape Town, South Africa; matsmith2@gmail.com \\ ${ }^{2}$ Astrophysics, Cosmology and Gravity Centre (ACGC), Department of Mathematics and Applied Mathematics, \\ University of Cape Town, Rondebosch 7701, South Africa \\ ${ }^{3}$ Institute of Cosmology and Gravitation, University of Portsmouth, Portsmouth PO1 3FX, UK \\ ${ }^{4}$ Las Cumbres Observatory Global Telescope Network, 6740 Cortona Dr., Suite 102, Goleta, CA 93117, USA \\ ${ }_{5}^{5}$ Department of Physics, University of California, Santa Barbara, Broida Hall, Mail Code 9530, Santa Barbara, CA 93106-9530, USA \\ ${ }^{6}$ Center for Particle Astrophysics, Fermilab, P.O. Box 500, Batavia, IL 60510, USA \\ ${ }^{7}$ Department of Astronomy \& Astrophysics, The University of Chicago, 5640 S. Ellis Ave, Chicago, IL 60637, USA \\ ${ }^{8}$ Kavli Institute for Cosmological Physics, The University of Chicago, 5640 S. Ellis Ave, Chicago, IL 60637, USA \\ ${ }^{9}$ African Institute for Mathematical Sciences, 6-8 Melrose Road, Muizenberg 7945, South Africa \\ ${ }^{10}$ South African Astronomical Observatory, P.O. Box 9, Observatory 7935, South Africa \\ ${ }^{11}$ Department of Mathematics and Applied Mathematics, University of Cape Town, Rondebosch 7701, South Africa \\ ${ }^{12}$ Department of Physics and Astronomy, Wayne State University, Detroit, MI 48201, USA \\ ${ }^{13}$ Department of Physics, University of Notre Dame, 225 Nieuwland Science Hall, Notre Dame, IN 46556, USA \\ ${ }^{14}$ Department of Physics and Astronomy, Rutgers, the State University of New Jersey, 136 Frelinghuysen Road, Piscataway, NJ 08854, USA \\ ${ }^{15}$ Physics \& Astronomy, University of Pennsylvania, 209 South 33rd Street, Philadelphia, PA 19104, USA \\ ${ }^{16}$ Department of Astronomy and Astrophysics, Pennsylvania State University, 525 Davey Laboratory, University Park, PA 16802, USA \\ ${ }^{17}$ Institute for Gravitation and the Cosmos, The Pennsylvania State University, University Park, PA 16802, USA \\ 18 The Oskar Klein Centre, Department of Astronomy, AlbaNova, Stockholm University, SE-106 91 Stockholm, Sweden \\ Received 2011 August 24; accepted 2012 June 7; published 2012 July 26
}

\begin{abstract}
Using data from the Sloan Digital Sky Supernova Survey-II (SDSS-II SN Survey), we measure the rate of Type Ia supernovae ( $\mathrm{SNe}$ Ia) as a function of galaxy properties at intermediate redshift. A sample of $342 \mathrm{SNe}$ Ia with $0.05<z<0.25$ is constructed. Using broadband photometry and redshifts, we use the PÉGASE.2 spectral energy distributions to estimate host galaxy stellar masses and recent star formation rates (SFRs). We find that the rate of $\mathrm{SNe}$ Ia per unit stellar mass is significantly higher (by a factor of $\sim 30$ ) in highly star-forming galaxies compared to passive galaxies. When parameterizing the $\mathrm{SN}$ Ia rate $\left(\mathrm{SNR}_{\mathrm{Ia}}\right)$ based on host galaxy properties, we find that the rate of SNe Ia in passive galaxies is not linearly proportional to the stellar mass; instead an $\mathrm{SNR}_{\mathrm{Ia}} \propto M^{0.68}$ is favored. However, such a parameterization does not describe the observed $\mathrm{SNR}_{\mathrm{Ia}}$ in star-forming galaxies. The $\mathrm{SNR}_{\mathrm{Ia}}$ in star-forming galaxies is well fitted by $\mathrm{SNR}_{\mathrm{Ia}}=(0.41 \pm 0.15) \times 10^{-10} M^{0.72 \pm 0.15}+(0.65 \pm 0.25) \times 10^{-3} \mathrm{SFR}^{1.01 \pm 0.22}$ (statistical errors only), where $M$ is the host galaxy stellar mass (in $M_{\odot}$ ) and SFR is the SFR (in $M_{\odot} \mathrm{yr}^{-1}$ ). We show that our results, for SNe Ia in passive galaxies, are consistent with those at higher redshifts (favoring $\mathrm{SNR}_{\mathrm{Ia}} \propto M$ ) when accounting for the difference in the ages of our galaxies. This suggests that the rate of SNe Ia is correlated with the age of the stellar population. The MLCS extinction parameter, $A_{V}$, is similar in passive and moderately star-forming galaxies, but we find indications that it is smaller, on average, in highly star-forming galaxies. This result appears to be driven by a deficit of the reddest $\left(A_{V}>0.15\right) \mathrm{SNe}$ Ia in highly star-forming galaxies. We consider that the high levels of dust in these systems may be obscuring the reddest and faintest SNe Ia.
\end{abstract}

Key words: cosmology: observations - distance scale - galaxies: evolution - supernovae: general

Online-only material: color figures, machine readable table

\section{INTRODUCTION}

Type Ia supernovae (SNe Ia) have been extensively studied because they provide accurate relative distances on cosmological scales. Measurements of SNe Ia have indicated that the expansion of the universe is currently accelerating (Kessler et al. 2009a; Lampeitl et al. 2010a; Conley et al. 2011; Sullivan et al. 2011; Suzuki et al. 2012), leading to the introduction of a "Dark Energy" component in our model of the universe.

$\mathrm{SNe}$ Ia are thought to arise from carbon-oxygen white dwarfs that accrete mass from a companion star and approach the Chandrasekhar mass limit, resulting in a thermonuclear explosion (Hoyle \& Fowler 1960; Branch et al. 1995; Yungelson \& Livio 1998). However, there is still significant debate on the details, e.g., the explosion mechanism, the accretion process, and the progenitor companion star, which may be a giant star, a main-sequence star, or a secondary white dwarf (Höflich et al. 2003). A measurement of the delay time (i.e., the time between the formation of the binary system and its thermonuclear explosion) constrains the possible progenitor systems (Greggio 2005). The delay time distribution (DTD) can be determined observationally by comparing the observed $\mathrm{SNe}$ Ia rates in galaxies with different star formation histories (SFHs; Gal-Yam \& Maoz 2004; Dahlen et al. 2004; Totani et al. 2008; Maoz et al. 2010; Maoz et al. 2011).

It has been observationally determined that $\mathrm{SNe}$ Ia are distinctly more common in galaxy hosts with recent star formation activity (Oemler \& Tinsley 1979). Recent work has determined that the SNe Ia rate per unit stellar mass $(\mathrm{SNuM})$ depends on host galaxy morphology and $(B-K)$ color (Mannucci et al. 
$2005)$ and that the $\mathrm{SN}$ Ia rate $\left(\mathrm{SNR}_{\mathrm{Ia}}\right)$ in late-type galaxies is a factor $\sim 20$ higher than in E/S0 galaxies. SNe Ia are seen locally to be rarer in galaxy bulges than spiral arms (Wang et al. 1997) and more common in blue galaxies than red (Mannucci et al. 2005). The population associated with star formation suggests that the $\mathrm{SNR}_{\mathrm{Ia}}$ contains a population with a cosmologically short time delay, while the observation of SNe Ia in very old systems indicates the existence of a population with large time delay (Cappellaro et al. 1999).

Mannucci et al. (2005) and later Scannapieco \& Bildsten (2005), Mannucci et al. (2006), and Sullivan et al. (2006) proposed a "two-component" $\mathrm{SNR}_{\mathrm{Ia}}$, consisting of a prompt component, dependent on recent host galaxy star formation, and a delayed component dependent on galaxy stellar mass. The overall $\mathrm{SNR}_{\mathrm{Ia}}$ is thus the sum of these two components, and can be further generalized as a function of the galaxy star formation rate (SFR) and stellar mass. Observations strongly favor a two-component model over a single-component model (Sullivan et al. 2006) and since the cosmic SFR increases with redshift, we expect that the prompt component will become a larger fraction of the $\mathrm{SN}$ Ia population with increasing redshift. Howell et al. (2007) show that the average light-curve width for normal SNe Ia increases by $8.1 \% \pm 2.7 \%$ between $z=0.03$ and 1.12 , consistent with any "prompt" component beginning to dominate at high redshift. Maoz et al. (2010), using cluster rate measurements, suggest a universal DTD, independent of environment and parameterized by $\mathrm{SNR}_{\mathrm{Ia}} \propto$ $t^{-1.2 \pm 0.3}$.

Several attempts have been made to constrain the functional form of the SNe Ia rate. Sullivan et al. (2006), using $124 \mathrm{SNe}$ Ia from the SNLS survey, found that, for passive galaxies, the $\mathrm{SNe}$ Ia rate is consistent with a linear relationship with host galaxy stellar mass. Recently, Li et al. (2011a) used a sample of 274 SNe Ia from the LOSS survey to consider how the size and morphology of the host galaxy affect the SNe Ia rate. They favor a power-law relationship between galaxy stellar mass and the SNuM with exponent approximately one-half independent of both galaxy morphology and color. Li et al. (2011b) find evidence for both a "prompt" (age < 420 Myr) and a "delayed" component ranging between 2.4 and $13 \mathrm{Gyr}$. Li et al. (2011a) also show that $\mathrm{SNR}_{\mathrm{Ia}}$ in young stellar populations may be strongly correlated with the rate of core-collapse SN. Brandt et al. (2010) analyze host galaxy spectra from the Sloan Digital Sky Supernova Survey-II (SDSS-II SN Survey), and find strong evidence of two progenitor channels each contributing equally to the $\mathrm{SNR}_{\mathrm{Ia}}$. The "prompt" channel has a delay time of $400 \mathrm{Myr}$ and is associated with high-stretch SNe Ia while the other has a delay time of $\sim 2.4 \mathrm{Gyr}$ and produces low-stretch SNe Ia.

An $\mathrm{SNR}_{\mathrm{Ia}}$ composed of two components may have ramifications when SNe Ia are used to determine cosmological parameters. It is likely that two distinct progenitor systems would contribute to the observed intrinsic scatter in the distances measured with $\mathrm{SNe}$ Ia. The relation between light-curve decline rate and peak luminosity for SNe Ia has been well tested (Phillips 1993; Sullivan et al. 2011), but the physics behind this relation and the details of the explosion mechanism are only partially understood (Kasen \& Woosley 2007). Better understanding of the nature of SNe Ia explosions and progenitor systems will aid in improving the accuracy of SN Ia distance measurements (Lampeitl et al. 2010b; Sullivan et al. 2010).

In this paper, we investigate the characteristics of the host galaxies of SNe Ia at intermediate redshift using the SDSS-II $\mathrm{SN}$ data set following the methodology described in Sullivan et al. (2006). The SDSS-II SN survey is ideally suited to this task because it provides the largest, unbiased data set currently available, with well-understood efficiency corrections, in a redshift range fully in the Hubble flow, but with high signal-tonoise $(\mathrm{S} / \mathrm{N})$ observations. Our host galaxies are well measured in several filter bands, allowing us to accurately estimate the galaxy's properties. The aim of this work is to measure the rate of SNe Ia explosions as a function of the host galaxy stellar mass and SFR. We parameterize the relationship for our intermediateredshift data, and interpret the results in the context of the twocomponent model.

This paper is organized as follows. In Section 2, we describe the SDSS-II SN Survey, the observing strategy and give a brief account of the results of this survey. In Section 3, we show how the SDSS-II SN data set is incomplete and introduce a method to produce an unbiased sample with a well-understood efficiency correction. In Section 4, we outline how the host galaxy of each SNe Ia is identified and the method that we use to determine the derived properties of the host galaxy. We also describe the sample of field galaxies used for comparison, and how it is corrected for incompleteness. In Section 6, we investigate how the $\mathrm{SNR}_{\mathrm{Ia}}$ is dependent on the properties of the host galaxy, studying how it is dependent on the stellar mass of the galaxy (Section 6.1), the SFR of the host galaxy (Section 6.3), and the specific star formation rate (sSFR) of the host (Section 6.5). Finally, Section 7 discusses how the light-curve shape (Section 7.1) and extinction (Section 7.2) of the SN Ia events are related to the galaxy host properties. Our conclusions are given in Section 8.

\section{THE SDSS-II SN SURVEY}

In this work, we use the full sample from the SDSS-II SN Survey (Frieman et al. 2008). This provides one of the largest samples of SNe Ia currently available.

The SDSS-II SN Survey was a three-year rolling search that produced a sample of spectroscopically confirmed $\mathrm{SNe}$ Ia with well-measured multi-color light curves at intermediate redshift $(z<0.4)$ using the SDSS $2.5 \mathrm{~m}$ telescope (York et al. 2000; Strauss et al. 2002; Gunn et al. 2006) at Apache Point Observatory with a wide-field CCD camera (Gunn et al. 1998). Observations were made in the SDSS ugriz filters (Fukugita et al. 1996), alternating between the northern and southern "strips" of the field designated as "Stripe 82" (Stoughton et al. 2002), bounded by $-60^{\circ}<\alpha(\mathrm{J} 2000)<60^{\circ}$, and $-1.258<\delta(\mathrm{J} 2000)<1.258$. Adverse weather and bright moonlight resulted in an average observation of each strip once every four nights with typical limiting magnitudes of $g \sim 21.8$, $r \sim 21.5, i \sim 21.2$ per observation. The scene modeling photometry (SMP) technique of Holtzman et al. (2008) was used to produce accurate photometric data for each $\mathrm{SN}$ event.

The SDSS-II SN Survey identified many thousands of transient events, of which 513 were spectroscopically confirmed as SNe Ia and 85 were other SN types (Sako et al. 2008; Zheng et al. 2008). Spectroscopic redshifts for the host galaxies of 339 probable SNe Ia, based on their light curves, were also obtained, and are discussed further in Section 3.

The first year SDSS-II SN sample was used for cosmological analyses (Kessler et al. 2009a; Sollerman et al. 2009; Lampeitl et al. 2010a). Dilday et al. (2008, 2010) measured the SNe Ia volumetric rate, Lampeitl et al. (2010b), Gupta et al. (2011), Konishi et al. (2011), and D'Andrea et al. (2011) analyzed the effect of host galaxies on light-curve parameters, both from the 
photometric properties of the host galaxies and studying their spectral features.

The SDSS-II SN Survey is approximately magnitude limited, producing an otherwise unbiased sample. This analysis uses a sample of $342 \mathrm{SNe}$ Ia in the redshift range $0.05<z<0.25$, where the efficiency of the survey is high (Dilday et al. 2010). This homogenous sample is comprised of 197 spectroscopically confirmed $\mathrm{SNe}$ Ia, with a further 87 having a host galaxy spectroscopic redshift. All objects are selected using a welldefined selection criteria, and have well-measured light curves that are consistent with an $\mathrm{SNe}$ Ia template, based on the Bayesian light-curve-fitting method of Sako et al. (2008). The selection criteria used to create this sample is discussed in Section 3.

\section{INCOMPLETENESS CORRECTIONS}

There are two major sources of inefficiency in the SDSS-II SN pipeline that lead to potential biases in the spectroscopically confirmed SN sample: detection efficiency and spectroscopic incompleteness. The detection efficiency was primarily magnitude limited and is amenable to calculation by simulation. The spectroscopic selection and analysis depends on many factors that are difficult to quantify. We adopt a strategy of augmenting the sample of spectroscopically confirmed SNe Ia with a sample of photometrically classified SNe Ia, identified by their lightcurve shape and color and correcting for detection efficiency.

\subsection{Correcting for Spectroscopic Incompleteness}

The SDSS-II SN Survey prioritized spectroscopic follow-up observations of SN candidates using a Bayesian classification method (Sako et al. 2008). ${ }^{19}$ However, the final ranking and decisions on spectroscopic follow-up priorities were based on the telescope's capabilities, local weather conditions, and the SN position on the sky, thus leading to a spectroscopic sample whose selection criteria are difficult to describe quantitatively. To produce a homogeneous sample of SN Ia candidates, we therefore seek a sample selection that avoids the uncertain and time-varying spectroscopic target selection process. We also seek a sample with high-quality light curves and low levels of contamination. However, we must also ensure that the majority of detected SNe Ia pass this criteria, so that our results are not dominated by the efficiency corrections.

We adopt a two-stage process. In the first stage, we use photometry obtained during the SN Ia search and the Bayesian classification method to apply very loose cuts that are intended to reduce the large number of non-SN Ia transient objects that are classified as candidates by the SDSS-II search pipeline, while retaining any $\mathrm{SN}$ Ia that could possibly survive our subsequent quality cuts. This sample is then analyzed by the more accurate SMP photometry and fit by the MLCS2k2 light-curve fitter to obtain a sample of probable SN Ia. The criteria used for our two-stage process is described as follows.

First, as part of the SDSS-II SN operations, every transient object with more than two epochs was selected to be a candidate, after known active galactic nuclei (AGNs), variable stars, and pipeline artifacts were removed. There are $\sim 20,000$ such candidates. The Bayesian classification technique, used in the SDSS-II SN search operations, fits SNe Ia, Ib/c, and II template light curves to each candidate, producing a probability, $p_{T}$, for

\footnotetext{
19 An updated version of this method is given in Sako et al. (2011). However, as our goal is to replicate the follow-up strategy of the SDSS-II SN Survey, it is not used in this work.
}

a candidate to belong to each class $(T)$ of SNe. This method assumes that each candidate is an SN of some particular type, but has been shown, nevertheless, to be accurate in differentiating between different SN types (Sako et al. 2008; Kessler et al. 2010). This Bayesian classification technique was applied to each candidate, and the following criteria was used to select viable $\mathrm{SN}$ Ia candidates.

1. At least three search discovery epochs.

2. $p_{\text {Ia }}>0.45$.

3. If the candidate has more than five search photometry epochs, the best-fit Ia model is not SN 2005gj. ${ }^{20}$

Additional cuts were considered, including using the photometric redshift from the nearest host galaxy to constrain the light curve, but were rejected, as it is significantly harder to model the SDSS-II SN survey selection function with those cuts. Our criteria select 1762 candidates, including $88 \%$ of the spectroscopically confirmed SNe Ia. Of the $12 \%$ of confirmed SNe Ia that fail this selection criteria, 27\% (17) were only observed on one or two occasions, $70 \%$ (45) do not satisfy the $p_{\text {Ia }}$ criteria, and $3 \%$ (2) are best fit by a 2005 gj-like template (including SN $2005 \mathrm{gj}$ itself).

The selection criteria described above use photometry obtained during the SN Ia search to produce a sample of candidates containing the vast majority of the spectroscopically confirmed SNe Ia, while removing the vast majority of non-SN Ia transient objects. In the second stage of our selection criteria, this sample was then analyzed using the more complete and more accurate SMP photometry and fit using the MLCS2k2 light-curve fitter (Jha et al. 2007; Kessler et al. 2009b) to ensure each candidate has a well-covered light curve and is well fit by an SN Ia event. The selection criteria are the same as were used by Kessler et al. (2009a) and Dilday et al. (2010), namely,

1. At least five photometric observations (all at different epochs) between -20 and +60 days relative to peak light in the rest frame of the $\mathrm{SN}$.

2. At least one epoch with $\mathrm{S} / \mathrm{N}>5$ in each of $g, r$, and $i$ (not necessarily the same epoch in each passband).

3. At least one photometric observation at least 2 days prior to maximum brightness in the $\mathrm{SN}$ rest frame.

4. At least one photometric observation at least 10 days past maximum brightness in the $\mathrm{SN}$ rest frame.

5. MLCS2k2 light-curve fit probability $>0.001 .^{21}$

6. MLCS2k2 light-curve decline rate parameter of $\Delta>$ $-0.4 .^{22}$

7. $-51^{\circ}<\alpha(\mathrm{J} 2000)<57^{\circ}$.

Excess color in SNe Ia is parameterized by MLCS2k2 using Cardelli et al. (1989), where $E(B-V)=A_{V} / R_{V}$. For this analysis, we assume an $A_{V}$ prior in the fitting process of $P\left(A_{V}\right)=e^{-A_{V} / \tau}$, with $\tau=0.33$, as described in Kessler et al. (2009a). We adopt a value of $R_{V}=2.3$ to match the best-fit result of Kessler et al. (2009a), but note that this value

\footnotetext{
20 SN 2005gj (Aldering et al. 2006; Prieto et al. 2007) is a peculiar SN, with a flat light curve after maximum. In addition to removing SN 2005gj-like SNe, this criterion also removes AGNs and other non-transient events from our sample.

21 Six of the spectroscopically confirmed SNe Ia fail this criteria, including four peculiar SNe Ia.

22 The cuts on MLCS2k2 light-curve fit probability and $\Delta(5,6)$ have a negligible effect on the size of our sample compared to the sampling cuts $(1,2,3,4)$.
} 
Table 1

Number of Candidates Passing Each Stage of Section 3.1

\begin{tabular}{lcc}
\hline \hline & No. of Candidates & Spectroscopically Confirmed \\
\hline All SDSS-II SN candidates & 19046 & 513 \\
After Bayesian light-curve fit & 1762 & 449 \\
Passing sample selection & 842 & 319 \\
$0.05<z<0.25$ & 379 & 217 \\
\hline
\end{tabular}

does not significantly affect our final sample or results. ${ }^{23}$ For comparison, $R_{V}=3.1$ on average for our galaxy, but previous SN Ia studies have favored values of $R_{V} \sim 2.0$ (Nobili \& Goobar 2008; Lampeitl et al. 2010b).

Of the 1762 candidates that satisfy the Bayesian light-curve fitter criteria, 842 satisfy the sample selection. Of these 842 , 319 are spectroscopically confirmed as SNe Ia and 180 are unconfirmed but have a host galaxy spectroscopic redshift.

The SNANA version (Kessler et al. 2009b) of MLCS is able to estimate a photometric redshift for SN candidates in addition to determining a distance modulus. Most of our 842 candidates lack spectroscopic redshift measurements, so we adopt a cosmological model of $\Omega_{M}=0.3$ and $\Omega_{\Lambda}=0.7$, to reduce the number of fit parameters, and to determine a photometric redshift for each candidate. To construct a sample that is unbiased with respect to spectroscopic followup, and has a well-determined selection function, we fit for a photometric redshift for all candidates, regardless of whether a spectroscopic redshift is known. An analysis of the accuracy of these photometric redshift estimates is given in Dilday et al. (2010), who find that the photometric redshifts are negligibly biased and are accurate to $\sim 0.01$ at low redshift $(0<z<0.25)$.

These selection criteria ensure that each candidate has a wellcovered light curve that is well fitted by a normal SN Ia event (peculiar SNe Ia will generally not pass the selection criteria).

While we have relied on photometric redshifts for the initial sample selection, we use spectroscopic redshift information, when available, for redshift selection and all subsequent analysis. To construct a sample that is primarily comprised of spectroscopically confirmed SNe Ia and not dominated by photometrically classified SNe Ia, and to avoid low detection efficiency (see Section 3.2), we restrict our SN sample to the redshift range $0.05<z<0.25$. This leaves $379 \mathrm{SNe}$ Ia. We find $217(57 \%)$ are spectroscopically confirmed SNe Ia, while $94(25 \%)$ are unconfirmed but have host galaxy spectroscopic redshifts and $68(18 \%)$ have no spectroscopic redshift information. The number of candidates that satisfy each stage of our selection criteria is shown in Table 1 . While the majority of our

\footnotetext{
23 Adopting a value of $R_{V}=2.1$ decreases the size of the sample that pass our sample selection from 842 to 830 , compared to 832 when $R_{V}=2.5$ is considered. However, we only consider SNe Ia with $0.05<z<0.25$, our sample size changes from 379 to 380 when both $R_{V}=2.1$ and 2.5 are considered.
}

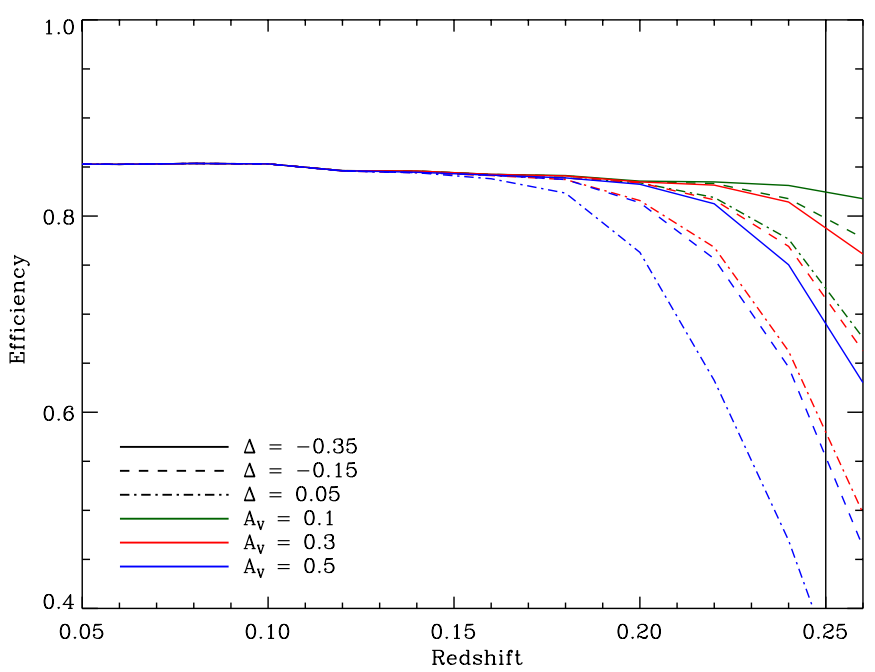

Figure 1. Efficiency of SN detection as a function of redshift for various values of $\Delta$ and $A_{V}$. The high-redshift limit used in this analysis is also shown.

(A color version of this figure is available in the online journal.)

sample has been spectroscopically confirmed, a significant fraction of candidates are only photometrically classified. However, Dilday et al. (2010) conservatively estimated that there is a $3 \%$ probability for non-SNe Ia to satisfy our selection criteria, and the total estimated contamination by non-SNe Ias is $2 \%$.

Table 2 lists the number of SNe Ia that pass our selection criteria for several redshift ranges, including the proportion of each sample that is spectroscopically confirmed as SNe Ia. As expected, the proportion of spectroscopically confirmed SNe decrease with increasing redshift, but it remains above $50 \%$ out to $z=0.25$.

\subsection{Determining the Survey Efficiency}

Having defined a homogeneous sample of SNe Ia candidates with $0.05<z<0.25$, we need to know the SDSS-II SN detection efficiency, $\epsilon(z)$. A detailed analysis of the efficiency was given in Dilday et al. (2008, 2010), differing here only in our determination of the efficiency as a function of $\Delta$ and $A_{V}$. Simulated SNe Ia, with a range of sky positions, time of peak brightness, redshifts, decline rate parameters, extinction and host galaxy position, and realistic errors were added directly to the image data and were processed by the SDSS-II SN pipeline (Sako et al. 2008) to obtain the efficiency as a function of $\mathrm{S} / \mathrm{N}$. SNANA was then used to determine the efficiency as a function of redshift, $\Delta$ and $A_{V}$, to allow for any variation in the intrinsic brightness of SNe Ia as a function of host galaxy type.

The proportion of SNe Ia that satisfy the criteria defined in Section 3.1 is shown as a function of redshift in Figure 1 for various values of $A_{V}$ and $\Delta$. For comparison, the sample of 379 SNe Ia defined previously has $\bar{\Delta}=-0.16$ and $\overline{A_{V}}=0.35$.

Table 2

Number of Candidate SNe Ia as a Function of Redshift

\begin{tabular}{|c|c|c|c|c|}
\hline Redshift Limit & Total & Spectroscopically Confirmed & Host Redshift & Photo-z Only \\
\hline $0.05<z<0.15$ & 88 & $73(83.0 \%)$ & $11(12.5 \%)$ & $4(4.5 \%)$ \\
\hline $0.05<z<0.25$ & 379 & $217(57.3 \%)$ & $94(24.8 \%)$ & $68(17.9 \%)$ \\
\hline $0.05<z<0.30$ & 559 & $272(48.7 \%)$ & $141(25.2 \%)$ & $146(26.1 \%)$ \\
\hline
\end{tabular}


We also highlight the high-redshift limit used in this analysis. All SNe Ia are detected with an efficiency of $>50 \%$, with the exception of SNe Ia with $A_{V} \geqslant 0.5$ and $\Delta \geqslant 0.05$. This is not surprising as these $\mathrm{SNe}$ Ia are also the faintest $\mathrm{SNe}$ Ia. The fact that the efficiency is less than $100 \%$ at low redshift is caused by $\mathrm{SN}$ explosions that occur late or early in the observing season and fail to allow the required number of observations. This inefficiency is a major effect at all redshifts but is accurately modeled in our simulation. The uncertainty on the survey efficiency is discussed in detail in Dilday et al. (2010).

We have now defined a uniformly selected sample of $379 \mathrm{SNe}$ Ia candidates with $0.05<z<0.25$ from the three years of the SDSS-II SN Survey, and calculated the efficiency of the survey in this redshift range as a function of $\Delta$ and $A_{V}$, which we shall invert to weight the galaxies in our sample. The uncertainty on the survey's efficiency is small (Dilday et al. 2010) compared to the statistical precision of our data and it is not necessary for us to include the uncertainty in our analysis. We now turn to consider the host galaxies of these $\mathrm{SN}$ events.

\section{HOST GALAXY DETERMINATION AND DERIVED QUANTITIES}

Here, we describe the method used to identify the host galaxies and determine their characteristics, such as stellar mass and recent SFR, for the $379 \mathrm{SNe}$ Ia identified in Section 3. We also outline the comparison field sample used to describe the underlying galaxy population in our redshift range.

\subsection{Host Galaxy Determination}

Repeat imaging of SDSS Stripe 82 has enabled the coaddition of images into a deep stacked image (Abazajian et al. 2009). The stack ranges from 20 to 40 individual images (depending on sky position) in all five SDSS filters (ugriz) and is roughly 2 mag deeper than a single epoch SDSS image. To determine the host galaxy for each SN in our sample, we match the SN positions with SDSS galaxies detected in this deep stacked image within a 0.25 arcmin radius. We require that the host galaxy has an SDSS model magnitude (Stoughton et al. 2002 ) in the range $15.5<r<23.0$ to ensure robust photometry. This magnitude cut is conservative, but applied to ensure that the SDSS-II SN pipeline is able to accurately distinguish between stars and galaxies in the deep stacks. The magnitude limits remove $10 \%$ of our $\mathrm{SNe}$ with either unobserved or too-faint hosts. We then visually scan each host galaxy, using images with and without the SN present to ensure that our host galaxy association is accurate. In six cases, at low redshift, where the host is extended or resolved into multiple objects, we select by hand a more likely object as the host galaxy. Of the 379 SNe Ia candidates identified in Section 3, 342 have a valid host galaxy identification, of which 197 (58\%) are spectroscopically confirmed to be SNe Ia, and $87(25 \%)$ are spectroscopically unconfirmed but have a host galaxy spectroscopic redshift. The remaining 58 objects are classified to be $\mathrm{SNe}$ Ia through their photometry alone. Of the 37 candidates that lack a valid host galaxy, $29(78 \%)$ have a host galaxy candidate with $r>23.0$ and $8(22 \%)$ have no host candidate within a 0.25 arcmin radius.

\subsection{Derived Host Galaxy Properties}

Having identified the host galaxy position and magnitudes for 342 SNe Ia candidates, we now determine their stellar mass and recent SFR.
There are several methods to infer galaxy properties from broadband photometry. A simple cut on the color of the galaxy can be used to infer its spectral type (Strateva et al. 2001) and the UV flux can provide an estimate of the recent SFR (Donas et al. 1987). These simple methods are able to differentiate between galaxies with markedly different levels of star formation activity, but struggle with galaxies with similar colors because multi-band photometry is not used (Baldry et al. 2006). Therefore, we fit our multi-band photometry to a set of spectral energy distributions (SEDs) and use the best-fit template to determine the galaxy parameters. This technique is widely used for photometric redshift estimates (Bolzonella et al. 2000; Le Borgne \& Rocca-Volmerange 2002; Oyaizu et al. 2008).

\subsubsection{SED Fitting}

The method used here is consistent with that of Sullivan et al. (2006), who studied the $\mathrm{SNR}_{\mathrm{Ia}}$ as a function of host galaxy properties at high redshift, allowing our results to be compared within the same framework. A discussion on how the different redshift ranges covered by this analysis and that of Sullivan et al. (2006) may affect our host galaxy derived properties is given in Appendix E.

We use the SEDs produced by the PÉGASE.2 galaxy spectral evolution code (Fioc \& Rocca-Volmerange 1997; Le Borgne et al. 2004). These templates have been used extensively in the literature to constrain the evolution of galaxies, particularly at high redshift (Glazebrook et al. 2004; Grazian et al. 2006). We use the set of eight evolutionary tracks listed in Table 1 of Le Borgne \& Rocca-Volmerange (2002) (excluding the starburst template), and assume a Kroupa (2001) initial mass function (IMF). In these scenarios, SFR is determined using the relationship SFR $=v \times M_{\text {gas }}$, where $v$ ranges from 0.07 to $3.33 \mathrm{Gyr}^{-1}$, and $M_{\text {gas }}$ is the density of gas in solar masses. Extinction due to dust is modeled internally, with a King (1980) profile used for the Elliptical template, and a plane-parallel slab geometry is used for the spiral and irregular templates. Each of the eight evolutionary scenarios is evolved over 69 time steps, each one corresponding to a different galaxy age, making a total of 552 template SEDs.

These SEDs are convolved with the SDSS filter responses (Fukugita et al. 1996) and fitted to the galaxy fluxes (calculated from model magnitudes after correcting for Galactic dust absorption from Schlegel et al. 1998 and AB system offsets) using the Z-PEG photometric redshift code (Le Borgne \& Rocca-Volmerange 2002). We keep the redshift of the SN host galaxies fixed to the spectroscopic redshift (from either the SN or host galaxy) or the photometric redshift determined by MLCS2k2. Applying a redshift constraint eliminates the color uncertainty due to the cosmological redshift. As dust is included internally in the SEDs, no dust correction is applied in the fitting process. We assume a default $\Lambda$ CDM cosmology $\left(\Omega_{M}=0.3, \Omega_{\Lambda}=0.7\right)$ and consider only templates that are younger than the age of the universe at the fitted redshift.

The best-fit template is determined through a $\chi^{2}$ minimization using all five SDSS filters. The total stellar mass of each galaxy is determined by integrating the SFH of the best-fitting SED and subtracting the mass of stars that have died. We characterize recent star formation with a mean SFR, since the instantaneous SFR is difficult to estimate without highresolution spectroscopic data. We use the result of Sullivan et al. (2006), who found that averaging the SFR over a period of $0.5 \mathrm{Gyr}$ can be accurately recovered by the PÉGASE. 2 
Table 3

List of SN Ia Events Used in This Paper and Their Associated Host Galaxy Properties

\begin{tabular}{|c|c|c|c|c|c|c|c|c|}
\hline \multicolumn{2}{|c|}{ Designation } & \multicolumn{2}{|c|}{ Host Position } & \multirow[t]{2}{*}{ Redshift } & \multirow{2}{*}{$\begin{array}{c}\text { Stellar Mass } \\
\qquad\left(\log M_{\odot}\right)\end{array}$} & \multirow{2}{*}{$\begin{array}{c}\mathrm{SFR}^{\mathrm{a}} \\
\left(\log M_{\odot} \mathrm{yr}^{-1}\right)\end{array}$} & \multirow{2}{*}{$\begin{array}{l}\mathrm{sSFR}^{\mathrm{a}} \\
\left(\mathrm{yr}^{-1}\right)\end{array}$} & \multirow[t]{2}{*}{$\mathrm{SN}^{\mathrm{b}}$} \\
\hline SN ID & IAU & $\alpha(J 2000)$ & $\delta(J 2000)$ & & & & & \\
\hline 762 & $2005 \mathrm{eg}$ & $01^{\mathrm{h}} 02^{\mathrm{m}} 08^{\mathrm{s}} .650$ & $-00^{\circ} 52^{\prime} 46^{\prime \prime} .766$ & $0.1915 \pm 0.0001$ & $11.02_{-0.18}^{+0.01}$ & $0.27<0.38<0.71$ & -10.64 & $\mathrm{sn}$ \\
\hline 779 & $\mathrm{~N} / \mathrm{A}$ & $01^{\mathrm{h}} 46^{\mathrm{m}} 41^{\mathrm{s}} .703$ & $-01^{\circ} 01^{\prime} 14^{\prime \prime} .270$ & $0.2377 \pm 0.0005$ & $10.10_{-0.01}^{+0.01}$ & $0.92<0.93<0.93$ & -9.18 & gal \\
\hline 822 & $\mathrm{~N} / \mathrm{A}$ & $02^{\mathrm{h}} 42^{\mathrm{m}} 14^{\mathrm{s}} .579$ & $-00^{\circ} 51^{\prime} 43^{\prime \prime} .607$ & $0.2166 \pm 0.0183$ & $9.85_{-0.19}^{+0.02}$ & $-99.00<-0.79<-0.46$ & -10.64 & lc \\
\hline 911 & $\mathrm{~N} / \mathrm{A}$ & $02^{\mathrm{h}} 34^{\mathrm{m}} 45^{\mathrm{s}} .829$ & $-00^{\circ} 06^{\prime} 54^{\prime \prime} .968$ & $0.2080 \pm 0.0100$ & $10.29_{-0.29}^{+0.07}$ & $0.59<0.80<0.81$ & -9.49 & gal \\
\hline 1008 & $2005 \mathrm{il}$ & $01^{\mathrm{h}} 53^{\mathrm{m}} 06^{\mathrm{s}} .704$ & $+01^{\circ} 06^{\prime} 49^{\prime \prime} 642$ & $0.2260 \pm 0.0100$ & $10.46_{-0.18}^{+0.02}$ & $-99.00<-0.18<0.15$ & -10.64 & gal \\
\hline
\end{tabular}

Notes.

${ }^{a}$ Passive galaxies are represented by -99.00 .

${ }^{\mathrm{b}}$ Redshift used in host galaxy template fitting based on SN spectra (sp), galaxy spectra (gal), or light curve (lc).

(This table is available in its entirety in a machine-readable form in the online journal. A portion is shown here for guidance regarding its form and content.)

SEDs, without introducing significant systematic uncertainties, especially for galaxies where the redshift is unknown.

Uncertainties in the galaxy properties are determined from the range spanned by the SEDs satisfying $\chi^{2} \leqslant \chi_{\min }^{2}+1$. We consider errors on the galaxy fluxes from the co-added image, with a minimum error as given in Blanton \& Roweis (2007). The stellar mass and recent SFR for the 342 host galaxies used in this analysis are given in Table 3.

\subsection{Comparison Field Sample}

To determine how our SN sample relates to the underlying galaxy population in our redshift range, we require a sample of galaxies that is representative of the general galaxy population. For this sample, we use galaxies detected in the deep stacks described in Section 4.1. We consider galaxies identified in the SDSS-II SN Survey region with $15.5<r<23.0$. Thus cut also removes the possibility of variable limiting magnitudes across the image.

We determine the stellar masses and recent SFRs for each galaxy in this sample using the same method as for the host galaxy sample except that the redshift is a free parameter to be determined by the Z-PEG fit. We require that the fitted redshift must lie in the redshift range $0<z<2$. The additional freedom allowed in determining the redshift for the field galaxies can result in large error bars on the derived photometric redshift, stellar mass, and SFR estimates. In extreme cases, there can be two or more distinct best-fit template solutions, resulting in more than one photometric redshift estimate and spectral type. In these cases, the galaxy is excluded from our analysis because the spectral classification and derived galaxy properties are ambiguous. To match the host galaxy population, we consider the $\sim 750,000$ galaxies with $0.05<z<0.25$.

\subsection{Correcting For Incompleteness in the Field Sample}

The comparison field sample is magnitude limited, and thus becomes increasingly incomplete at higher redshifts, with only the brightest galaxies observed at higher redshifts. Galaxies with a given absolute magnitude (and spectral type) will pass the apparent magnitude selection criteria $(15.5<r<23.0)$ at different redshifts, which may be less than the full survey range $(0.05<z<0.25)$. To correct for this effect, we use the $V_{\max }$ method (Schmidt 1968; Felten 1976). Using the best-fitting SED for each field galaxy, we calculate its absolute magnitude and $k$-correction, and determine the redshift limits at which it would satisfy $15.5<r<23.0$. Whenever the redshift range is less than the total survey range $(0.05<z<0.25)$, we weight the galaxy by $V_{\text {survey }} / V_{\max }$, where $V_{\max }$ is the comoving volume for which each galaxy will remain within our survey's magnitude limits, and $V_{\text {survey }}$ is the comoving volume of the SDSS-II SN survey, i.e., for a redshift range, $0.05<z<0.25$ and constant for each galaxy in our sample. Eighty-three percent of the field galaxies in our sample have redshift limits larger than that of the SDSS-II SN survey, and are not affected by this correction. The remaining $17 \%$ of field galaxies are on average weighted by a value of 4.98. Since this form of incompleteness will affect both the comparison field sample and host galaxy sample, this incompleteness correction is applied to both, although only 3 of the 342 host galaxies in our sample are affected by this correction.

\subsection{Systematic Uncertainties}

Systematic uncertainties in our derived galaxy properties can arise from many sources including the wavelength coverage of the SDSS filters, our decision to use the PÉGASE.2 SEDs, our choice of IMF, the accuracy of the photometric redshifts for the comparison field sample, the accuracy of the PÉGASE.2 stellar mass estimates, and the ability of PÉGASE.2 to accurately recover the stellar masses and SFRs for a sample of simulated galaxies. All these systematic errors are discussed further in Appendices A-E.

In Appendix A, we show that the PÉGASE. 2 SEDs primarily use the color of a galaxy as a proxy to infer its spectral type. The reddest galaxies are classified as passive galaxies, with the bluest galaxies considered highly star forming. Moderately star-forming galaxies are distributed between passive and highly star-forming galaxies, spanning a large range of color.

In Appendix B, we investigate the accuracy of the PÉGASE.2 photometric redshift estimates for our field sample. We find a mean offset in redshift of $\Delta z=0.03$, with the photometric redshift estimate being smaller than the known spectroscopic redshift. This redshift error results in an error in stellar mass of $\Delta \log M=0.22 M_{\odot}$. In Appendix $\mathrm{C}$, we show the effect that applying this offset to our data would have on the results presented in Section 6 and show that they are consistent. This offset provides us with an estimate of our systematic uncertainty, but due to a lack of understanding of the cause of this offset, it is not applied to our nominal analysis.

Appendix D studies how the stellar mass and SFR estimates from PÉGASE. 2 for our host galaxy sample compare to those determined using the spectral features of galaxies. We consider a 


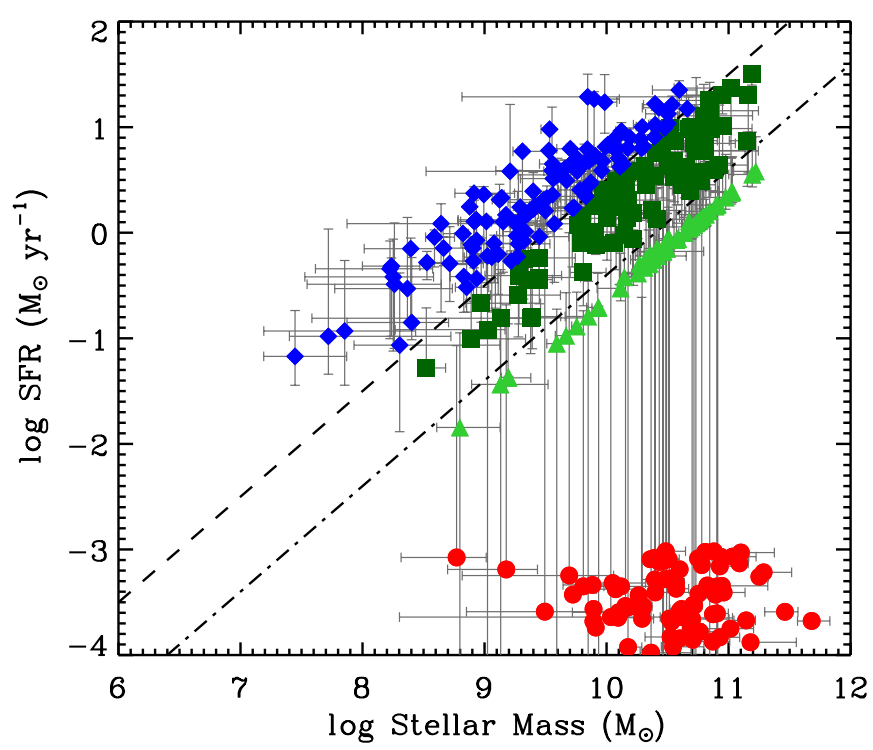

Figure 2. Distribution of stellar mass and SFR for the 342 SN host galaxies. Highly star-forming galaxies are shown as blue diamonds and passive galaxies as red circles. Moderately star-forming galaxies are plotted in green, with light green triangles indicating the "ridge line" of galaxies discussed in the text and Appendix A, and the remaining population plotted as dark green squares. The dash-dotted line highlights this split. Passive galaxies have SFR $=0$ but are shown here as randomly distributed in the range $-4<\log \mathrm{SFR}<-3$. The dashed line indicates the split used to distinguish highly star-forming galaxies from those with moderate levels of star formation activity.

(A color version of this figure is available in the online journal.)

sample of SDSS galaxies that have spectroscopically measured stellar masses and SFRs (Brinchmann et al. 2004; Kauffmann et al. 2003) and compare these to estimates determined in our analysis. We find that the stellar masses are recovered, with no significant offset, but there is a mean offset in the SFR of $\Delta \log \mathrm{SFR}=0.12 M_{\odot} \mathrm{yr}^{-1}$. However, Brinchmann et al. (2004) measure the "instantaneous" (present-day) SFR instead of our "recent" SFR, which is averaged over the last $0.5 \mathrm{Gyr}$, so the two quantities are not directly comparable.

In Appendix E, we consider how the rest-wavelength coverage of the SDSS filter set affects our stellar mass estimates. With increasing redshift, the SDSS filters will sample a different rest-wavelength range. This can be particularly important for systems with a variety of stellar populations, such as merging galaxies. To examine the sensitivity to our wavelength coverage, we repeat the determination of stellar masses and SFRs using only three or four of the five SDSS filters. We find an increased scatter in the results, but no overall bias in the stellar mass or SFR estimates. This is particularly encouraging, because it suggests that the comparison of our galaxy properties with those of Sullivan et al. (2006) will not be affected by the different cosmological redshifts of the two surveys.

\section{HOST GALAXY PROPERTIES}

In Section 3, we defined a sample of homogeneously selected SNe Ia, and in Section 4, determined a host galaxy for each object. Having estimated their stellar mass and recent SFR, we now analyze these derived properties and how they relate to the $\mathrm{SN}$ rate.

Figure 2 shows the distribution of our host galaxy sample in stellar mass and SFR. Galaxies are shown in three categories, highly star forming (blue), moderately star forming (green), and passive (red). The highly and moderately star-forming galaxies are separated by their sSFR: the SFR per unit stellar mass (Guzman et al. 1997; Brinchmann \& Ellis 2000; Brinchmann et al. 2004; Sullivan et al. 2006). We followed Sullivan et al. (2006) in choosing $\log \mathrm{sSFR}=-9.5$ as the arbitrary division between highly and moderately star forming as indicated by the dashed line in Figure 2. Highly star-forming galaxies are using a significant proportion of their stellar mass to form new stars and their stellar populations are expected to be dominated by young, massive stars. Galaxies classified as moderately star forming are likely to be dominated by an older, more evolved population of stars. Passive galaxies have a nominal SFR $=0$, but for display purposes, are randomly distributed in red in Figure 2 around $\log \mathrm{SFR}=-3.5$. The average stellar mass of a passive galaxy is $\log M=10.52 M_{\odot}$, considerably more massive than starforming galaxies, which average $\log M=9.91 M_{\odot}$, consistent with other observations of the local universe (Taylor et al. 2009).

Of the 342 galaxies in our sample, $80(23 \%)$ are classified as passive galaxies, $139(41 \%)$ have moderate levels of star formation activity, and $123(36 \%)$ are highly star forming.

In Figure 2, we note a "ridge line" of galaxies, which is classified as moderately star forming, but has the lowest possible values of sSFR allowed. A dash-dotted line is shown in Figure 2 to highlight this population of galaxies. Seventy-eight percent of these galaxies are best fit by the lenticular S0 (scenario), with the remaining $22 \%$ being best described by the elliptical template. In comparison $52 \%$ of the remaining moderately star-forming galaxies are best fit by the $\mathrm{S} 0$ scenario. In Appendix A, we show the color-magnitude diagram, and conclude that these galaxies lie at the edge of the distribution of the moderately star-forming galaxies but appear to be distinct from the passive galaxies. Thus, we do not remove these galaxies from our analysis. We will show later through a Monte Carlo (MC) approach that removing these galaxies from our sample does not affect our major conclusions. These galaxies lie on a boundary in the PÉGASE. 2 templates, which is not preferentially occupied by the comparison field sample, potentially due to the redshift of these galaxies being left as a free parameter in the Z-PEG fit.

While we determine the SFRs for the host galaxies in our sample by integrating over the last $0.5 \mathrm{Gyr}$ of the $\mathrm{SFH}$, to match the analysis of Sullivan et al. (2006), several recent studies (Sullivan et al. 2010) use a period of 0.25 Gyr. To determine the effect that this has on the classification of our host galaxies, we recalculate the SFRs for our sample of galaxies by integrating over a period of $0.25 \mathrm{Gyr}$. We find that the number of passive galaxies is unaffected by this change, while the mean $\log (\mathrm{SFR})$ is 0.11 higher when $0.5 \mathrm{Gyr}$ is integrated over to determine the SFR. 44.4\% (152) of the sample is initially categorized as moderately star forming when a period of $0.25 \mathrm{Gyr}$ is considered. As the division between moderately and highly star-forming galaxies is arbitrary, we find that by choosing $\log \mathrm{sSFR}=-9.58$ to separate the two samples, we produce a sample of 124 galaxies that are classified as highly star forming, of which only $4(3 \%)$ were not originally classified as highly star forming. Using a period of $0.25 \mathrm{Gyr}$ to determine the SFR still results in an identical "ridge line" of galaxies, discussed above. We thus infer that the period of the SFH integrated over to determine the SFR does not significantly affect our classification.

\section{SN Ia RATE}

We now turn to looking at how the SN rate depends on the galaxy properties of total stellar mass and recent star formation for passive and star-forming galaxies. 


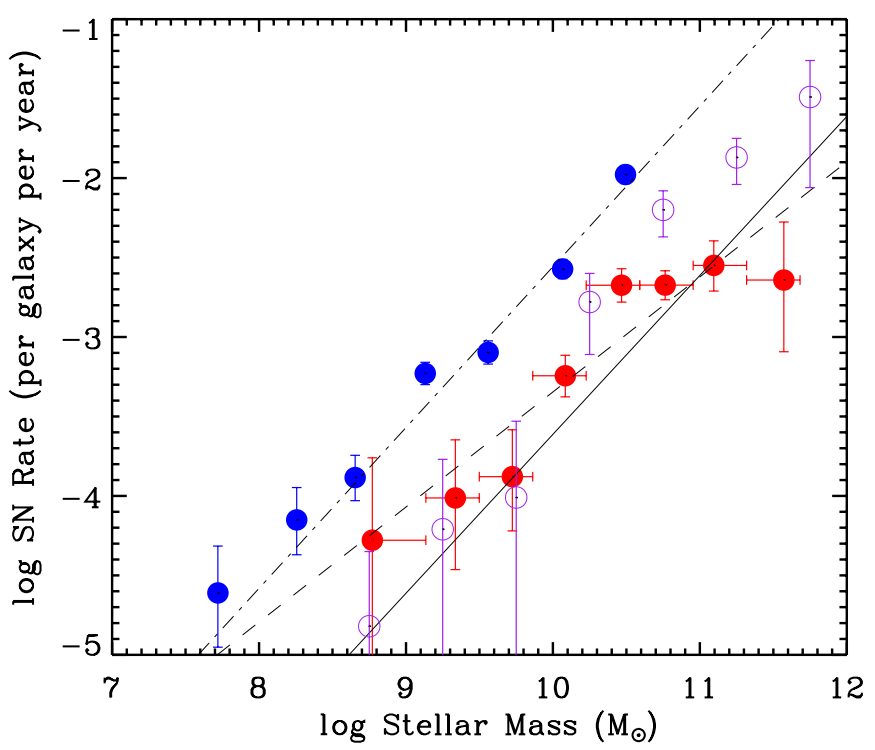

Figure 3. SN Ia rate as a function of host galaxy stellar mass. The values for star-forming (passive) galaxies are shown in blue (red). The data points, for passive galaxies, from Sullivan et al. (2006) are shown as open circles. The best-fitting lines for passive and star-forming galaxies are shown as dashed and dot-dashed lines, respectively. Also shown is a fit (solid line) to the passive galaxies where the line slope is assumed to be one.

(A color version of this figure is available in the online journal.)

\subsection{SN Ia Rate as a Function of Host Galaxy Stellar Mass}

According to the standard model of galaxy formation, passive galaxies are primarily comprised of old, low-mass stellar systems that evolve without forming new stars. It is reasonable to suppose that the SN Ia population in passive galaxies could only occur as a result of a process with a delay time that is long compared to the age of the galaxy. If that is the case, then the number of SN Ias occurring in these environments could be expected to be proportional to the host galaxy stellar mass. On the other hand, if the delay time is only comparable to the age of the galaxy, there could be a more complicated dependence based on the details of the SFH.

To measure the stellar mass dependence with the SDSS data, we split both our host galaxy sample and comparison field sample into passive and star-forming galaxies. The samples are binned by their stellar mass, with both the host galaxy and field sample weighted for incompleteness using the $1 / V_{\max }$ correction, and the efficiency correction applied to the host galaxy sample. Each host galaxy is weighted by $1 / \epsilon$, the survey efficiency at the redshift of the SNe given the year it was observed. The efficiency correction ranges between 1.4 and 2.6, with a mean weighting of 1.9 for each host galaxy. By dividing the number of host galaxies by the corresponding number of field galaxies, and including a correction for the survey's observing period, we can determine how the rate of $\mathrm{SNe}$ Ia varies as a function of the stellar mass of their host galaxy. Figure 3 shows the $\mathrm{SNR}_{\mathrm{Ia}}$ for both the passive and star-forming galaxy samples. It is clear that the rate of $\mathrm{SNe}$ in all types of galaxies depends on the stellar mass. We also see that the relationship between the $\mathrm{SNR}_{\mathrm{Ia}}$ and stellar mass is different for passive galaxies and star-forming galaxies in the SDSS data.

The data are fit to a linear function in log-space, corresponding to a power-law dependence of $\mathrm{SN}$ rate on stellar mass as shown in Figure 3. A linear dependence on stellar mass would result in a slope of unity. The error bars shown and fitting errors include statistical errors only and are determined using the effective number of objects in each bin, such that $\sigma=$ $\left(\left(\sum \mathrm{eff}^{-2}\right) \times n_{\mathrm{gal}}\right)^{1 / 2}$, where eff is the determined efficiency for each SNe Ia. The uncertainty in galaxy stellar mass is discussed in Section 6.7. For passive galaxies, we find a best-fit slope of $0.72 \pm 0.13$ (with $\chi^{2}$-statistic $\left(\chi^{2}\right)=5.65$ for 6 degrees of freedom) compared to a value of $1.01 \pm 0.09\left(\chi^{2}=15.65\right.$ for 6 degrees of freedom, denoted as $n_{\text {star forming }}$ ) for star-forming galaxies. The value for passive galaxies is incompatible (at the $2.1 \sigma$ level) with a linear relationship, as favored by Sullivan et al. (2006), who found a slope of $1.10 \pm 0.12$ using the SNLS data at higher redshift.

Figure 3 also shows the results for passive galaxies from Sullivan et al. (2006) as open circles. We see that the SDSS galaxy sample contains fewer SNe Ia in high stellar mass passive galaxies than SNLS and more SNe Ia in low stellar mass passive systems. While the two analyses should be directly comparable, the galaxy population is expected to evolve between $z \sim 0.75$ and $z \sim 0.2$. In addition, we note that the SDSS analysis finds a larger slope for star-forming galaxies compared to passives, while the opposite is seen in the SNLS data, who find values of $n_{\text {star forming }}=0.66 \pm 0.08$ and $0.74 \pm 0.08$, for moderately and highly star-forming galaxies, respectively. Li et al. (2011a) find that the slope is independent of host galaxy type, with a value of 0.5 providing a good fit in all cases.

A physical understanding of why the SNe Ia rate in passive galaxies is not linearly proportional to stellar mass is unclear. One explanation is that the SNe Ia rate may be driven by the age of the stellar population, such that the SNe Ia DTD is proportional to $t^{-1}$. Maoz et al. (2011) found that the $\mathrm{SNR}_{\mathrm{Ia}}$ decreases monotonically with the age of the stellar population, with the oldest galaxies (age $>2.4 \mathrm{Gyr}$ ) having an $\mathrm{SNR}_{\mathrm{Ia}}$ significantly lower than younger (age $<420$ Myr) stellar populations. If low-mass stellar systems have a younger average age for the stellar populations, they would have a higher rate of $\mathrm{SNe}$ Ia per unit stellar mass.

In this case, to better compare with the results of Sullivan et al. (2006), we select a galaxy sample that has the same distribution of stellar age. Wake et al. (2006) showed that there is no evidence that luminous red galaxies are evolving beyond passive evolution between $0<z<0.6$. The stellar mass is proportional to the age of a galaxy at a fixed redshift (Thomas et al. 2010). Therefore, the stellar population for a galaxy of fixed stellar mass will be older at $z=0.2$ compared to $z=0.75$. To consider this possibility, we restrict our passive galaxy sample to $M_{\text {stellar }}<11.3$ to correct for the redshift evolution from $z=0.75$ to $z=0.2$, and recalculate our best-fitting slope for passive galaxies. We find a slope of $0.90 \pm 0.20$, consistent with a linear relationship.

This result suggests that the SNe Ia rate is related to the mean stellar age of the population. The most massive galaxies have a deficit of SNe Ia compared to their lower mass counterparts at a fixed redshift, due to their older stellar population. However, this result does not affect the observed differences in star-forming galaxies, where the SFH is considerably more complex.

An alternative conclusion may be that this analysis assumes that a Kroupa IMF is a reasonable approximation for both low- and high-mass galaxies. Should this assumption not be valid, and the IMF in high-mass galaxies differs from that of low-mass galaxies, then this would explain the apparent difference in the $\mathrm{SNe}$ Ia rate in high- and low-mass galaxies. 


\subsection{Parameterizing the SN Ia Rate}

The data in Figure 3 indicate that the $\mathrm{SNR}_{\mathrm{Ia}}$ depends on galaxy stellar mass, but also that the rate depends on whether the galaxy is actively forming stars. A two-component model was considered by Mannucci et al. (2006), and Scannapieco \& Bildsten (2005), who modeled the $\mathrm{SNR}_{\mathrm{Ia}}$ of a galaxy to consist of a "delayed" component, with a long delay time that is driven by the stellar mass of the galaxy, and a "prompt" component, with short delay times that is caused by the formation of new stars. The model assumes that the "delayed" component is proportional to the stellar mass independent of the galaxy age and SFH, and that the "prompt" component timescale is short compared to changes in the SFR. These assumptions result in an expression whose parameters can be determined from data as was done by Sullivan et al. (2006). In detail, the $\mathrm{SNR}_{\mathrm{Ia}}$ can be written as

$$
\operatorname{SNR}_{\mathrm{Ia}}(t)=A \times M(t)+B \times \operatorname{SFR}(t),
$$

where $\operatorname{SNR}_{\mathrm{Ia}}(t)$ is the explosion rate of SNe Ia at time $t, M(t)$ is the stellar mass of a galaxy, $\operatorname{SFR}(t)$ is the rate of change of stellar mass, and $A$ and $B$ are constants determined from the

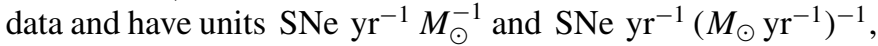
respectively. $\operatorname{SFR}(t)$ is averaged over the previous $0.5 \mathrm{Gyr}$ as discussed in Section 4.2. While the model is, in principle, valid for all $t$, our SN rate measurements apply only to the current era and we will suppress the dependence on $t$. This model is commonly known as the "A+B" model for the $\mathrm{SN}$ rate and assumes that the $\mathrm{SNR}_{\mathrm{Ia}}$ is linearly dependent on both the stellar mass of a galaxy and its SFR. However, in Section 6.1 we showed that for passive galaxies (whose $\mathrm{SNR}_{\mathrm{Ia}}$ will be purely dependent on stellar mass in this parameterization), a linear dependence was not favored by the SDSS data set. We therefore generalize Equation (1) to

$$
\mathrm{SNR}_{\mathrm{Ia}}=A \times M^{n_{\mathrm{M}}}+B \times \mathrm{SFR}^{n_{\mathrm{SFR}}},
$$

where $n_{\mathrm{M}}, n_{\mathrm{SFR}}, A$, and $B$ are constants to be determined from the data. Since passive galaxies have $\mathrm{SFR}=0$, we can apply the results of Section 6.1 to conclude $n_{\mathrm{M}}=0.72 \pm 0.13$. The straight line fit to the passive galaxies yields $\log A=$ $-10.59 \pm 0.63$ or $A=0.26_{-0.20}^{+0.83} \times 10^{-10} \mathrm{SNe} \mathrm{yr}^{-1} M_{\odot}^{-1}$. If we assume $n_{\mathrm{M}} \equiv 1$, we find a value of $\log A=-13.61 \pm 0.09$ or $A=2.44_{-0.45}^{+0.55} \times 10^{-14}$, which differs at $2.3 \sigma$ with the value of $A=5.3 \pm 1.1 \times 10^{-14}$ found using the SNLS data set.

To better interpret these results, compare them to previous analyses, and determine the $\mathrm{SNR}_{\mathrm{Ia}}$ due to stellar mass, we can determine the value of $\mathrm{SNR}_{\mathrm{Ia}}$ (Stellar Mass) $=A \times M^{n_{\mathrm{M}}}$ in Equation (2), at a fixed stellar mass, here chosen to be $1 \times 10^{10} M_{\odot}$. Considering $n_{\mathrm{M}}=0.72 \pm 0.13$, we find $\mathrm{SNR}_{\mathrm{Ia}}($ Stellar Mass $)=4.12 \times 10^{-4} \mathrm{SNe} \mathrm{yr}^{-1}$, compared to a value of $2.44 \times 10^{-4}$ when $n_{\mathrm{M}}=1$ is enforced. For comparison, Sullivan et al. (2006) found $5.3 \times 10^{-4}$ using the SNLS data set. These results are entirely consistent when errors on $A$ and $n_{\mathrm{M}}$ are considered.

While the above parameterization of the $\mathrm{SNR}_{\mathrm{Ia}}$ uses the stellar mass and the recent SFR, other galaxy properties can be considered, such as the metallicity, age, and level of extinction. Gallagher et al. (2005) find qualitative evidence suggesting that the progenitor age is a possible source of diversity in $\mathrm{SNe} \mathrm{Ia}$ properties. However, there is a degeneracy between the age of a galaxy, and its metallicity, which is extremely difficult to break using broadband photometry. We thus confine ourselves to considering the stellar mass and SFRs of our host galaxies in this analysis, but note that with improved stellar population models, a larger wavelength coverage, and galaxy spectra, it may be possible to break this degeneracy. Using SDSS-II SNe, Gupta et al. (2011) attempt to break this degeneracy by using multiwavelength photometry to better constrain the ages of their SN Ia host galaxies while D'Andrea et al. (2011) and Konishi et al. (2011) use spectral features to determine the metallicities of their host galaxies.

\subsection{SN Ia Rate as a Function of Host Galaxy Mean Star Formation Rate}

We now consider the star-forming galaxies to determine $B$ and $n_{\mathrm{SFR}}$. We bin the host galaxy and comparison field sample in SFR, and as in Section 6.1, correct both samples for incompleteness, using the SN efficiency for the host galaxy sample and the $1 / V_{\max }$ correction for both the host galaxy and comparison field samples. The $\mathrm{SNR}_{\mathrm{Ia}}$ is shown (blue diamonds) as a function of SFR in Figure 4. We want to determine the excess $\mathrm{SNR}_{\mathrm{Ia}}$ due to recent star formation activity assuming that the term proportional to stellar mass is the same for starforming and passive galaxies. The portion due to the stellar mass term is calculated using Equation (2), and shown in the figures

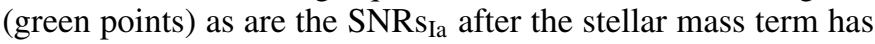
been subtracted (red points). The left panel of Figure 4 uses our best-fit line with slope $n_{\mathrm{M}}=0.72$ while the right panel uses the fit where the slope is fixed at $n_{\mathrm{M}} \equiv 1$.

The observed $\mathrm{SNR}_{\mathrm{Ia}}$ depends strongly on recent star formation and greatly exceeds the rate in passive galaxies with identical stellar mass. We find $n_{\mathrm{SFR}}=0.95 \pm 0.07$ and $\log B=$ $-2.91 \pm 0.05\left(B=1.23_{-0.12}^{+0.14} \times 10^{-3} \mathrm{SNe} \mathrm{yr}^{-1}\left(M_{\odot} \mathrm{yr}^{-1}\right)^{-1}\right)$ with $\chi^{2}=15.96$ for 6 degrees of freedom when $n_{\mathrm{M}}=$ 0.72 . When $n_{\mathrm{M}} \equiv 1$ is assumed, we find $n_{\mathrm{SFR}}=0.99 \pm$ 0.08 and $\log B=-2.94 \pm 0.05\left(B=1.16_{-0.13}^{+0.15} \times\right.$ $\left.10^{-3} \mathrm{SNe} \mathrm{yr}^{-1}\left(M_{\odot} \mathrm{yr}^{-1}\right)^{-1}\right)$ with $\chi^{2}=11.53$ for 6 degrees of freedom. The lack of sensitivity to the value of $n_{\mathrm{M}}=0.72$ follows because the stellar mass term is always small compared to the star-forming term.

Our best fit to Equation (2) is therefore

$$
\begin{aligned}
\mathrm{SNR}_{\mathrm{Ia}}= & 0.26_{-0.20}^{+0.83} \times 10^{-10} M^{0.72 \pm 0.13} \\
& +1.23_{-0.12}^{+0.14} \times 10^{-3} \mathrm{SFR}^{0.95 \pm 0.07}
\end{aligned}
$$

As noted previously, the analysis of Sullivan et al. (2006) in the redshift range $0.2<z<0.75$ preferred an $\mathrm{SNR}_{\mathrm{Ia}}$ linearly dependent to the stellar mass of a galaxy. If we assume $n_{\mathrm{M}} \equiv 1$ and $n_{\mathrm{SFR}} \equiv 1$, we find

$$
\mathrm{SNR}_{\mathrm{Ia}}=2.44_{-0.45}^{+0.55} \times 10^{-14} M+1.15_{-0.12}^{+0.13} \times 10^{-3} \mathrm{SFR} .
$$

For comparison, Sullivan et al. (2006) find values of $A=$ $(5.3 \pm 1.1) \times 10^{-14} \mathrm{SNe} \mathrm{yr}^{-1} M_{\odot}^{-1}$ and $B=(3.9 \pm 0.7) \times$ $10^{-4} \mathrm{SNe} \mathrm{yr}^{-1}\left(M_{\odot} \mathrm{yr}^{-1}\right)^{-1}$. Our value of $A$ is $2.3 \sigma$ lower, while the values of $B$ are inconsistent at $5.4 \sigma$, indicating that recent star formation activity plays a more significant role in determining the overall $\mathrm{SNe}$ Ia rate for our sample. This result is consistent with models of how galaxies evolve through cosmic time. Observations suggest, that at high redshift $(z=0.75)$, the rate of star formation is far higher than in the local universe. Combining this with measurements suggesting that the $\mathrm{SNR}_{\mathrm{Ia}}$ increases slowly as a function of redshift suggests that recent star formation activity is more significant in determining the 

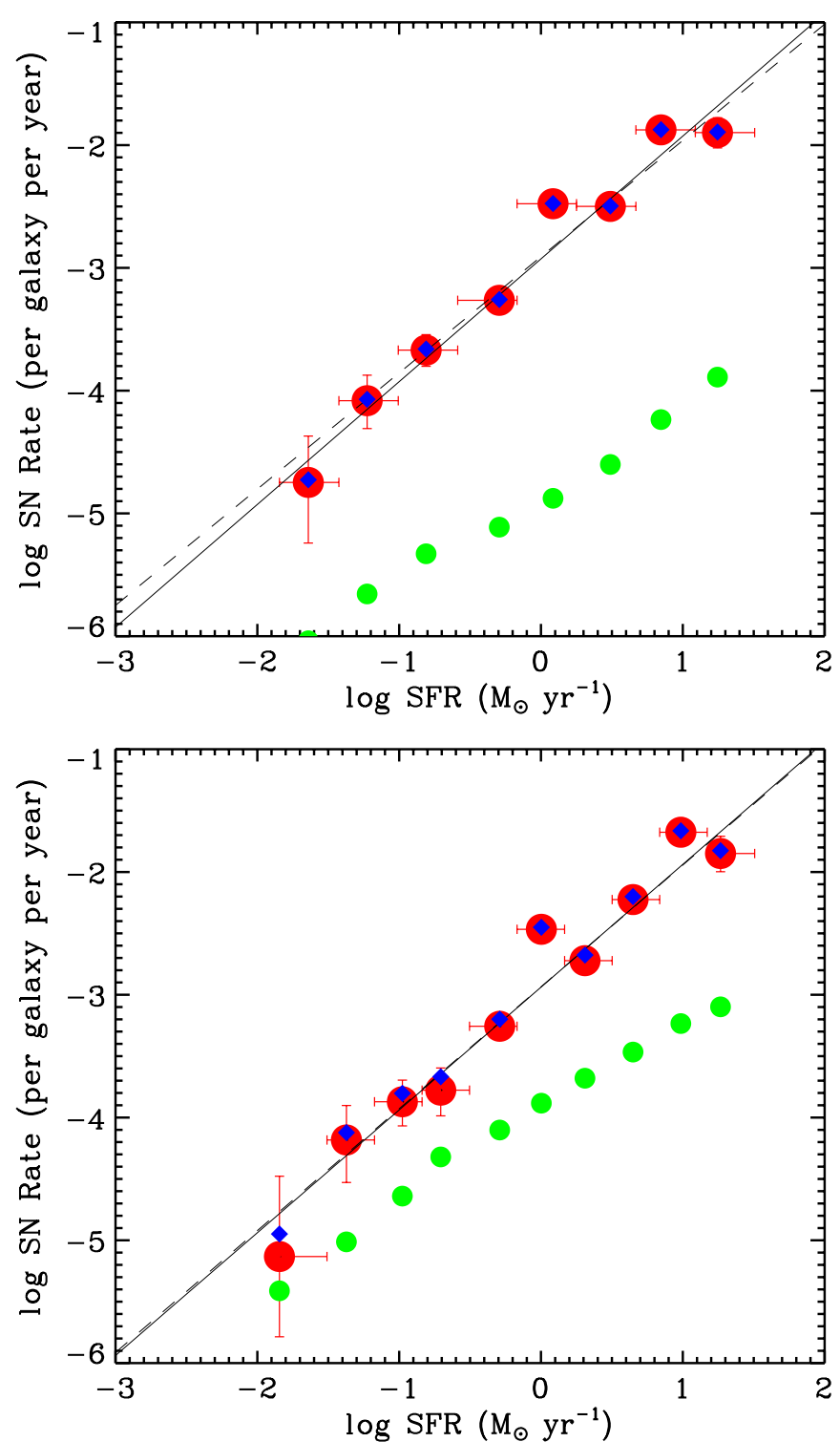

Figure 4. SN Ia rate as a function of host galaxy star formation rate. Left panel: green points indicate the expected rate of SNe Ia due to the stellar mass of each galaxy, using the values of $n_{\mathrm{M}}$ and $A$ as determined in Section 6.1. Blue diamonds show the observed rate of SNe Ia per galaxy per year, while the red points are the excess (i.e., the difference between the blue and green values). A best-fitting line (dashed) and best-fitting line with unit slope (solid) are also shown. Right panel: identical, except a value of $n_{\mathrm{M}} \equiv 1$ is assumed.

(A color version of this figure is available in the online journal.)

$\mathrm{SNR}_{\mathrm{Ia}}$ at low redshift. The methodology used in this analysis is similar to that used in Sullivan et al. (2006), and has been significantly tested (Appendices A, B, C, and E).

\subsection{Bivariate Fitting}

Thus far we have used only the passive galaxies to determine the $A$ term, and then used the star-forming galaxies to determine the $B$ term, while keeping $A$ fixed. A more sophisticated method is to constrain the parameters simultaneously using all galaxy types, thus making optimal use of the data. We bin the host galaxy and comparison field sample in the stellar mass and star formation plane, and correct for incompleteness. By dividing the number of host galaxies in each bin by the corresponding number of field galaxies, we are able to determine the $\mathrm{SNR}_{\mathrm{Ia}}$ in each bin of stellar mass and SFR. We consider several variations in Equation (2). First, we consider the case where $B \equiv 0$, i.e., the $\mathrm{SNR}_{\mathrm{Ia}}$ is purely dependent on stellar mass, and $n_{\mathrm{M}}$ is a free parameter. In this case, we find $A=(0.59 \pm 0.20) \times 10^{-10}$ and $n_{\mathrm{M}}=0.61 \pm 0.12$, with $\mathrm{SNR}_{\mathrm{Ia}}($ Stellar Mass $)=0.74 \times 10^{-4}$, in agreement with the result found in Section 6.1. However, this is a poor fit to the data, and allowing $B \neq 0$ but assuming $n_{\mathrm{SFR}} \equiv 1$ reduces the $\chi^{2}$ from 432 for 42 degrees of freedom to 325 for 41 degrees of freedom, and yields values of $n_{\mathrm{M}}, A$, and $B$ consistent with those found in Section 6.3. Finally, we allow $n_{\mathrm{SFR}}$ to vary and find $n_{\mathrm{SFR}}=1.01 \pm 0.22$ with $\chi^{2}=325$ for 40 degrees of freedom, a negligible improvement.

We thus conclude that our data are consistent with a linear dependence on SFR. Our fiducial result using bivariate fitting is

$$
\begin{aligned}
\mathrm{SNR}_{\mathrm{Ia}}= & (0.41 \pm 0.15) \times 10^{-10} M^{0.72 \pm 0.15} \\
& +(0.65 \pm 0.25) \times 10^{-3} \mathrm{SFR}^{1.01 \pm 0.22} .
\end{aligned}
$$

This is in good agreement with the values found in Sections 6.1 and 6.3 .

\subsection{SN Ia Rate as a Function of Specific Star Formation Rate}

The results from Sections 6.1, 6.3, and 6.4 have shown that the $\mathrm{SNR}_{\mathrm{Ia}}$ depends on both the galaxy stellar mass and SFR, with SFR dominating the $\mathrm{SNR}_{\mathrm{Ia}}$. Here, we study how the $\mathrm{SNR}_{\mathrm{Ia}}$ is related to host galaxy type. To determine this, we bin the host galaxy and comparison field samples according to their value of sSFR. Both samples are corrected for incompleteness, and the total stellar mass of the field sample is calculated. By dividing the incompleteness corrected number of host galaxies by the total stellar mass of the field sample, we are able to determine the $\mathrm{SNR}_{\mathrm{Ia}}$ per unit stellar mass as a function of sSFR. As noted in Section 5, sSFR is a way of distinguishing between galaxy types, with galaxies with low values of sSFR being primarily large galaxies that are using a small fraction of their total stellar mass to form new stars, while those with larger levels of sSFR are starburst galaxies, or galaxies that are using a significant fraction of their stellar mass to form new stellar systems.

Figure 5 shows the rate of SNe Ia per unit stellar mass in starforming galaxies as a function of sSFR. The SNe Ia rate per unit mass is a factor of $\sim 30$ times higher for starburst galaxies compared to passive galaxies. The SDSS data, however, has a point that appears to disagree with the other data and the generally linear trend of increasing $\mathrm{SNR}_{\mathrm{Ia}}$ with $\mathrm{SSFR}$. This point corresponds to the galaxies highlighted in Section 5 as being a population of "ridge-line" galaxies on the edge of the moderately star-forming galaxies, but which are not classified as passive. Appendix A considers these objects, and determines that while there was a possible ambiguity in the classification of these objects, they constitute a distinct population that lies between passive and star-forming galaxies. However, while these galaxies lie on a boundary of the PÉGASE. 2 templates, comparatively few field galaxies occupy the same bin, potentially due to the redshift of these galaxies being determined simultaneously in the Z-PEG fit. The exaggerated number of host galaxies in this bin, in combination with a low total stellar mass of the comparison field sample, results in an anomalously high $\mathrm{SNe}$ Ia rate per unit mass, compared to the results of Mannucci et al. (2005) and Sullivan et al. (2006). Due to the systematic uncertainty of this measurement, it is highlighted separately in Figure 5. Excluding this bin from our analysis, the rate increases with SSFR, and the measurements from this work are in agreement with those at higher redshift and in the local universe. 


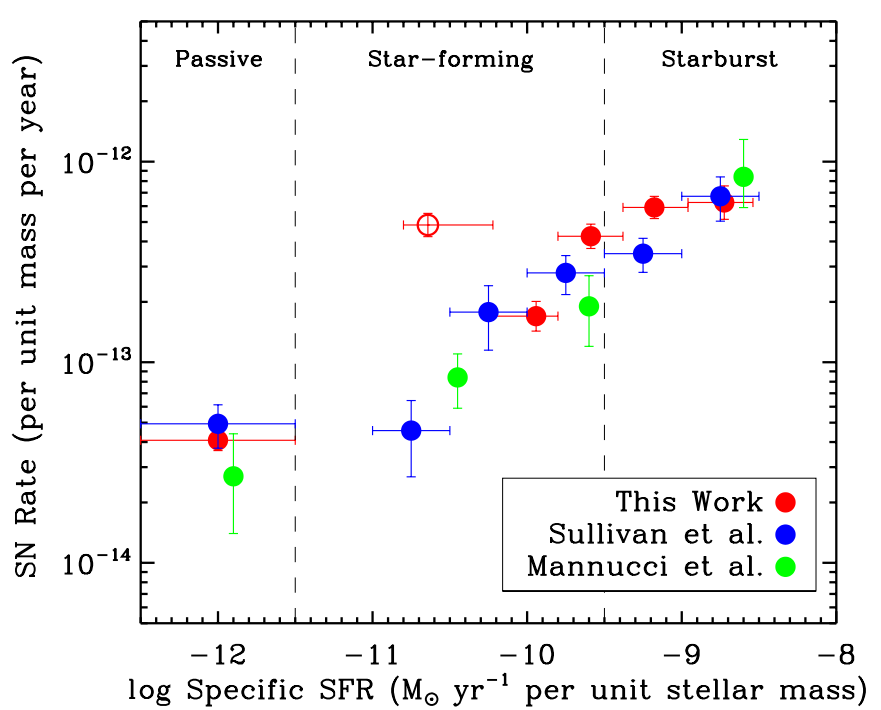

Figure 5. SN Ia rate per unit stellar mass per year as a function of host galaxy specific star formation rate (sSFR). The red points are those determined by the SDSS analysis, blue points are those from Sullivan et al. (2006), while points shown in green are measurements at low redshift made by Mannucci et al. (2005), where the magnitude and color of the host galaxy have been used to determine the host galaxy stellar mass and SFR. The horizontal errors on the SDSS data indicate the bin width while the horizontal positions represent the mean of the data in that bin. The positioning of green points on the $x$-axis is somewhat uncertain since precise values for sSFR were not given. Passive galaxies have $\mathrm{sSFR}=0$, but are shown on this graph, with $\log \mathrm{sSFR} \simeq-12$. The SDSS measurement contaminated by the systematically uncertain "ridge-line" galaxies (see the text in Section 5) is shown as an open circle for clarity.

(A color version of this figure is available in the online journal.)

\subsection{The Effect of Our Selection Criteria}

We have studied how the $\mathrm{SNR}_{\mathrm{Ia}}$ is related to the host galaxy properties for the SDSS sample. However, as discussed in Section 3.1, the sample constructed for this analysis is comprised of SNe Ia that have not all been spectroscopically confirmed and thus may be contaminated by non-SN Ia events. Our analysis has also used an efficiency correction, which is increasingly important toward the edge of our redshift range, and thus can cause uncertainties in our results.

Table 4 shows the results that we obtain using various subsets of our SN Ia sample. In the two left-hand columns, we show fits for the spectroscopically confirmed and unconfirmed portions of our sample. In the three rightmost columns, we show the results for three different redshift ranges. The spectroscopically confirmed and unconfirmed subsamples are, of course, incomplete, so the $A$ and $B$ parameters will necessarily be smaller than for the full sample. The results for $n_{\mathrm{M}}$ and $n_{\mathrm{SFR}}$, however, should be comparable.

From Table 4, we see that the spectroscopically confirmed sample is fit by $n_{\mathrm{M}}=0.84 \pm 0.24$, consistent with the combined result but also consistent with $n_{\mathrm{M}}=1$. This value of $n_{\mathrm{M}}$ may be due to the lower proportion of passive galaxies in this sample and a bias against more luminous and thus massive galaxies in the spectroscopic selection. This bias is caused by a targeting against probable $\mathrm{SNe}$ Ia that occur in the centers of luminous galaxies, making them difficult to identify spectroscopically. As the redshift limit considered is decreased, resulting in a more complete sample, the value of $n_{\mathrm{M}}$ is stable and shows no trend toward one (although the errors increase rapidly as the sample size is reduced). The value for $\log A$ when we assume $n_{\mathrm{M}} \equiv 1$ is consistent for all the redshift ranges (when $n_{\mathrm{M}}$ is a free parameter, it is highly degenerate with $\log A$ ). Table 4 also shows that the value of $n_{\mathrm{SFR}}$ is not influenced by the inclusion of non-spectroscopically confirmed $\mathrm{SNe}$ Ia nor the redshift range.

Table 4 also shows how our selection criteria affects the dependence that the $\mathrm{SNR}_{\mathrm{Ia}}$ has on the SFR. We showed in Sections 6.3 and 6.4 , that the $\mathrm{SNR}_{\mathrm{Ia}}$ depends approximately linearly on the recent SFR. The subsamples displayed in Table 4 are all consistent and there is no hint on any deviation from $n_{\mathrm{SFR}} \sim 1$. A value of $\log B \sim-2.85$ is valid for all redshift ranges considered.

Finally, we study the results of Section 6.5. The "passive rate" in Table 4 is the rate per unit stellar mass per year in passive galaxies, as shown in Figure 5, while the "starburst rate is the corresponding rate for galaxies with the highest levels of sSFR (sSFR > -9.5). The values of both the passive and starburst rates are consistent independent of the redshift limit used and are consistent with the sum of the spectroscopically confirmed and unconfirmed samples. In all cases, the rate of SNe Ia per unit stellar mass in highly star-forming galaxies is significantly higher (by a factor of $\sim 30$ ) than that seen in passive galaxies.

The fit parameters shown in Table 4 do not evolve across our redshift range. Since any evolution would be unexpected due to the small range in cosmic time covered by our analysis, it is reassuring to note that our results are insensitive to the redshift interval that is chosen. The only parameter that significantly changes with redshift is the proportion of passive galaxies found. This may simply reflect observations that $\mathrm{SNe}$ Ia in passive galaxies are fainter than their star-forming counterparts (see Section 7 for an analysis with the SDSS sample) and thus are not observed at higher redshifts by the SDSS-II SN survey.

In Table 4, we considered separately the spectroscopically confirmed and unconfirmed SNe Ia and various redshift ranges in our conclusions. However, there are several other uncertainties that can arise as part of our selection criteria and analysis. We also investigated the effect of using different priors on $A_{V}$ when determining the sample of $\mathrm{SNe}$ Ia, by using a flat prior and a positive prior $\left(A_{V} \geqslant 0\right)$. The various priors produce results that are entirely consistent with those found previously. In the determination of $n_{\mathrm{M}}$ and $n_{\mathrm{SFR}}$, we have performed linear fits to the log-log plots, but it is also possible to fit to the power-law form directly. These fits are consistent with our linear fits to the logarithms. We have also considered various bin sizes for each stage of our analysis and find that our results are unaffected. We also considered the possibility that our results may depend on a specific, anomalous year with the SDSS-II SN survey or may vary as a function of position on the sky. We split the host galaxy sample by both year and position but found no variation on our final results. Finally, we considered the effect of modeling $\epsilon$, the survey efficiency, as a function of redshift, $A_{V}$ and $\Delta$, to account for the observation that passive galaxies host fainter, higher $\Delta$, SNe than star-forming galaxies (Section 7), which could result in the survey efficiency varying as a function of host galaxy type. This additional correction, which has not been applied for other previous analyses, produces results that are entirely consistent with our fiducial result.

In order to study the robustness of our results, we have considered the effect of altering our selection criteria in Table 4. We have shown that the inclusion of non-spectroscopically confirmed SNe Ia in our sample and varying our redshift range considered does not significantly change the values of $A, B, n_{\mathrm{M}}$, and $n_{\mathrm{SFR}}$. 
Table 4

Effect of Our Selection Criteria on the Results Described in Sections 6.1, 6.3, and 6.5

\begin{tabular}{|c|c|c|c|c|c|c|}
\hline Parameter & Nominal Result & Confirmed $^{\mathrm{g}}$ & Phot-ID ${ }^{h}$ & $z<0.20$ & $z<0.16$ & $z<0.12$ \\
\hline No. hosts & 342 & 197 & 145 & 196 & 103 & 36 \\
\hline$\%$ Confirmed SNe & 57.6 & 100.0 & 0.0 & 67.9 & 79.6 & 97.2 \\
\hline$n_{\mathrm{M}}$ & $0.72 \pm 0.13$ & $0.84 \pm 0.24$ & $0.51 \pm 0.29$ & $0.68 \pm 0.17$ & $0.61 \pm 0.26$ & $0.57 \pm 0.89$ \\
\hline$n_{\text {star forming }}$ & $1.01 \pm 0.09$ & $0.92 \pm 0.10$ & $1.14 \pm 0.15$ & $0.96 \pm 0.11$ & $0.84 \pm 0.14$ & $0.86 \pm 0.28$ \\
\hline $\log A^{\mathrm{a}}$ & $-10.59 \pm 0.63$ & $-12.10 \pm 0.84$ & $-8.42 \pm 0.91$ & $-10.27 \pm 0.72$ & $-9.54 \pm 0.87$ & $-8.97 \pm 1.35$ \\
\hline $\log A^{\mathrm{b}}$ & $-13.61 \pm 0.09$ & $-13.85 \pm 0.12$ & $-13.74 \pm 0.12$ & $-13.74 \pm 0.12$ & $-13.73 \pm 0.14$ & $-13.51 \pm 0.24$ \\
\hline$n_{\mathrm{SFR}}{ }^{\mathrm{a}}$ & $0.95 \pm 0.07$ & $0.92 \pm 0.10$ & $0.90 \pm 0.15$ & $0.97 \pm 0.10$ & $1.00 \pm 0.11$ & $1.14 \pm 0.29$ \\
\hline$n_{\mathrm{SFR}}^{\mathrm{b}}$ & $0.99 \pm 0.08$ & $1.00 \pm 0.12$ & $0.95 \pm 0.20$ & $1.02 \pm 0.12$ & $1.06 \pm 0.16$ & $1.10 \pm 0.46$ \\
\hline $\log B^{\mathrm{c}}$ & $-2.91 \pm 0.05$ & $-3.24 \pm 0.06$ & $-3.27 \pm 0.08$ & $-2.98 \pm 0.06$ & $-3.04 \pm 0.08$ & $-3.02 \pm 0.15$ \\
\hline $\log B^{\mathrm{d}}$ & $-2.93 \pm 0.04$ & $-3.27 \pm 0.05$ & $-3.30 \pm 0.07$ & $-2.99 \pm 0.06$ & $-3.04 \pm 0.08$ & $-3.06 \pm 0.15$ \\
\hline $\log B^{\mathrm{e}}$ & $-2.97 \pm 0.05$ & $-3.29 \pm 0.06$ & $-3.41 \pm 0.10$ & $-3.03 \pm 0.07$ & $-3.15 \pm 0.10$ & $-3.17 \pm 0.18$ \\
\hline$\%$ Passive galaxies & 23.4 & 20.3 & 27.6 & 25.0 & 28.2 & 33.3 \\
\hline Passive rate ${ }^{f}$ & $4.1 \pm 0.7$ & $1.9 \pm 0.5$ & $2.2 \pm 0.5$ & $3.0 \pm 0.7$ & $2.3 \pm 0.7$ & $2.9 \pm 1.4$ \\
\hline Starburst rate ${ }^{\mathrm{f}}$ & $64.8 \pm 19.08$ & $42.3 \pm 15.5$ & $22.4 \pm 12.6$ & $87.1 \pm 41.4$ & $70.9 \pm 50.8$ & $87.6 \pm 81.0$ \\
\hline
\end{tabular}

Notes.

${ }^{\mathrm{a}} n_{\mathrm{M}}$ free, in units of $\mathrm{SNe} \mathrm{yr}^{-1} M_{\odot}^{-1}$.

${ }^{\mathrm{b}} n_{\mathrm{M}} \equiv 1$, in units of $\mathrm{SNe} \mathrm{yr}^{-1} M_{\odot}^{-1}$.

${ }^{\mathrm{c}} n_{\mathrm{M}}$ and $n_{\mathrm{SFR}}$ free, SNe $\mathrm{yr}^{-1}\left(M_{\odot} \mathrm{yr}^{-1}\right)^{-1}$.

${ }^{\mathrm{d}} n_{\mathrm{M}}$ free and $n_{\mathrm{SFR}} \equiv 1, \mathrm{SNe} \mathrm{yr}^{-1}\left(M_{\odot} \mathrm{yr}^{-1}\right)^{-1}$.

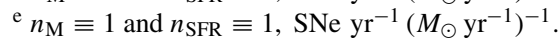

$\mathrm{f} \times 10^{-14}$ per unit stellar mass per year.

g Considering solely spectroscopically confirmed SN Ia.

${ }^{\mathrm{h}}$ Considering solely photometrically typed SN Ia.

\subsection{The Effect of SED Errors on Our Results}

For each host galaxy in our sample, we have determined a value for its stellar mass and recent SFR. Each of these measurements has an associated error that may allow galaxies to move between bins, and thus affect our fitted parameters. This may be especially important for the sample of galaxies with ambiguous classification, highlighted in Section 5 and Appendix A.

To quantify this effect on our results, we use both an MC and a Bootstrap (BP) approach. For the MC analysis, 10,000 realizations of the host galaxy sample are made by drawing from the estimated probability distribution for each host. We consider two cases: varying the stellar mass of each host galaxy and varying the stellar mass and SFR. The second case allows galaxies to move from passive to star forming and vice versa. Thus each of the MC samples consists of 342 host galaxies, each a variation on one specific host in the host galaxy sample.

For the BP analysis, we again obtain 10,000 realizations of the host galaxy sample, this time by selecting a host galaxy at random with replacement. This analysis allows each host galaxy to be selected on multiple occasions, probing the effect that outliers within the sample may have on our results. As with the MC approach we consider two cases: selecting the host galaxies before the sample has been separated into passive and star-forming data sets (thus allowing the relative proportions to change) and randomly sampling after separation, thus enforcing the same proportion of passive and star-forming galaxies in each data set. The second approach tests the dependence of our results on a subset of objects, while the first case is particularly important for the sample of ambiguous galaxies, identified in Section 5, and investigates if they are likely to be predominantly passive in nature.

To determine how the SED uncertainties affect our overall conclusions, we determine the value of each parameter for each realization, and fit a Gaussian (which is observed to provide a good fit) to each distribution. This provides an estimate for the central values and systematic uncertainty in each case. Table 5 gives the values and associated errors for the parameters determined in this work for each of these four systematic tests.

Both the MC and BP analysis provide values for the parameters determined that are consistent with those found as our main result, as described in Sections 6.1, 6.3, and 6.5. In all cases considered the observed scatter from the MC and BP tests is smaller than the statistical uncertainty. We note that while the central value for $n_{\mathrm{M}}$ determined by the MC analysis is larger than our default result, it is still inconsistent with $n_{\mathrm{M}}=1$ at the $3.4 \sigma$ level when the stellar mass is allowed to vary, and $2.9 \sigma$ when galaxies are allowed to move from passive to star forming.

We observe that the $\mathrm{SNR}_{\mathrm{Ia}}$ per unit stellar mass in passive galaxies is consistent in all four cases considered. This implies that the sample of galaxies with ambiguous classifications (as noted in Section 5) do not affect our overall conclusions. In the MC where the stellar mass and SFR are allowed to vary, these galaxies are able to move from moderately star forming to passive where their error bars allow. However, we note that in this case, the $\mathrm{SNR}_{\mathrm{Ia}}$ in passive galaxies is in fact lower than the observed value, suggesting that these galaxies are not passively evolving, and may have non-zero SFRs.

The systematic error bars determined by the MC and BP tests are sub-dominant to the statistical uncertainties obtained in Sections 6.1, 6.3, and 6.5. Therefore, the uncertainties due to the SED fitting are not the major source of uncertainty. Extensive testing of the PÉGASE.2 SEDs is carried out in Appendices A, B, D, and E. An offset is found in the photometric redshift estimates and associated stellar mass estimates for the comparison field sample used in our analysis. By assuming that this offset does not affect the SFRs for our galaxies, which are inferred through their color (which we determine in Appendix A), we uniformly apply this offset to each galaxy in the comparison field sample, and recalculate the results of Sections 6.1, 6.3, and 6.5. While we find some variation in the central values, our conclusions are unaffected. We find that 
Table 5

Effect of Our SED Uncertainty on the Results Described in Sections 6.1, 6.3, and 6.5

\begin{tabular}{|c|c|c|c|c|c|}
\hline Parameter & Nominal Result & MC (Variable M) & MC (Variable M and SFR) & BP (Split) & BP (Not Split) \\
\hline$n_{\mathrm{M}}$ & $0.72 \pm 0.13$ & $0.82 \pm 0.08$ & $0.82 \pm 0.08$ & $0.73 \pm 0.11$ & $0.73 \pm 0.11$ \\
\hline$n_{\text {star forming }}$ & $1.01 \pm 0.09$ & $1.01 \pm 0.08$ & $1.01 \pm 0.08$ & $1.00 \pm 0.11$ & $1.00 \pm 0.12$ \\
\hline $\log A^{\mathrm{a}}$ & $-10.59 \pm 0.63$ & $-11.62 \pm 0.85$ & $-11.64 \pm 1.00$ & $-10.72 \pm 1.15$ & $-10.72 \pm 1.16$ \\
\hline $\log A^{\mathrm{b}}$ & $-13.61 \pm 0.09$ & $-13.52 \pm 0.05$ & $-13.54 \pm 0.06$ & $-13.58 \pm 0.10$ & $-13.59 \pm 0.11$ \\
\hline$n_{\mathrm{SFR}}{ }^{\mathrm{a}}$ & $0.95 \pm 0.07$ & $0.95 \pm 0.003$ & $0.83 \pm 0.11$ & $0.96 \pm 0.07$ & $0.96 \pm 0.07$ \\
\hline$n_{\mathrm{SFR}}{ }^{\mathrm{b}}$ & $0.99 \pm 0.08$ & $0.96 \pm 0.003$ & $0.83 \pm 0.09$ & $0.98 \pm 0.07$ & $0.98 \pm 0.07$ \\
\hline $\log B^{c}$ & $-2.91 \pm 0.05$ & $-2.91 \pm 0.005$ & $-2.95 \pm 0.07$ & $-2.93 \pm 0.04$ & $-2.93 \pm 0.04$ \\
\hline $\log B^{\mathrm{d}}$ & $-2.93 \pm 0.04$ & $-2.93 \pm 0.006$ & $-2.99 \pm 0.11$ & $-2.94 \pm 0.04$ & $-2.95 \pm 0.04$ \\
\hline $\log B^{\mathrm{e}}$ & $-2.97 \pm 0.05$ & $-2.97 \pm 0.006$ & $-3.05 \pm 0.11$ & $-3.00 \pm 0.04$ & $-3.00 \pm 0.04$ \\
\hline Passive rate $\mathrm{f}^{\mathrm{f}}$ & $4.1 \pm 0.7$ & $3.5 \pm 0.01$ & $3.9 \pm 0.2$ & $3.8 \pm 0.01$ & $4.0 \pm 0.8$ \\
\hline Starburst rate $\mathrm{f}^{\mathrm{f}}$ & $64.8 \pm 19.08$ & $61.1 \pm 8.7$ & $56.9 \pm 9.3$ & $64.8 \pm 12.0$ & $65.3 \pm 12.3$ \\
\hline
\end{tabular}

Notes.

${ }^{\mathrm{a}} n_{\mathrm{M}}$ free, in units of $\mathrm{SNe} \mathrm{yr}^{-1} M_{\odot}^{-1}$.

${ }^{\mathrm{b}} n_{\mathrm{M}} \equiv 1$, in units of SNe $\mathrm{yr}^{-1} M_{\odot}^{-1}$.

${ }^{\mathrm{c}} n_{\mathrm{M}}$ and $n_{\mathrm{SFR}}$ free, SNe $\mathrm{yr}^{-1}\left(M_{\odot} \mathrm{yr}^{-1}\right)^{-1}$.

${ }^{\mathrm{d}} n_{\mathrm{M}}$ free and $n_{\mathrm{SFR}} \equiv 1, \mathrm{SNe} \mathrm{yr}^{-1}\left(M_{\odot} \mathrm{yr}^{-1}\right)^{-1}$.

${ }^{\mathrm{e}} n_{\mathrm{M}} \equiv 1$ and $n_{\mathrm{SFR}} \equiv 1, \mathrm{SNe} \mathrm{yr}^{-1}\left(M_{\odot} \mathrm{yr}^{-1}\right)^{-1}$.

${ }^{\mathrm{f}} \times 10^{-14}$ per unit stellar mass per year.

Table 6

A Comparison of the Results of Sections 6.1 and 6.3 from This Paper with Other Published Analyses

\begin{tabular}{|c|c|c|c|c|c|c|}
\hline Analysis & $\begin{array}{l}\text { Redshift Range } \\
\text { Covered }\end{array}$ & $n_{\mathrm{M}}^{\mathrm{a}}$ & $n_{\mathrm{SFR}}{ }^{\mathrm{b}}$ & $\begin{array}{c}A^{\mathrm{a}} \\
\left(\mathrm{SNe} \mathrm{yr}^{-1} M_{\odot}^{-1}\right)\end{array}$ & $B^{\mathrm{b}}$ & $\begin{array}{c}\mathrm{SNR}_{\mathrm{Ia}}{ }^{\mathrm{c}} \\
\text { (Stellar Mass) }\end{array}$ \\
\hline This work & $0.05<z<0.25$ & $0.72 \pm 0.15$ & $1.01 \pm 0.22$ & $0.41 \pm 0.15 \times 10^{-10}$ & $0.65 \pm 0.25$ & 2.38 \\
\hline This work & $0.05<z<0.25$ & fixed $=1$ & fixed $=1$ & $2.44_{-0.45}^{+0.55} \times 10^{-14}$ & $1.23_{-0.12}^{+0.14}$ & 2.44 \\
\hline Sullivan et al. (2006) & $0.2<z<0.75$ & $1.10 \pm 0.12$ & $0.84 \pm 0.06$ & - & - & - \\
\hline Sullivan et al. (2006) & $0.2<z<0.75$ & Fixed $=1$ & Fixed $=1$ & $5.3 \pm 1.1 \times 10^{-14}$ & $0.39 \pm 0.07$ & 5.3 \\
\hline Mannucci et al. $(2005)^{\mathrm{d}, \mathrm{e}}$ & Low redshift & Fixed $=1$ & Fixed $=1$ & $3.83_{-1.2}^{+1.4} \times 10^{-14}$ & $2.3 \pm 1$ & 3.83 \\
\hline Dilday et al. $(2008)^{\mathrm{f}}$ & $z<0.12$ & Fixed $=1$ & Fixed $=1$ & $2.8 \pm 1.2 \times 10^{-14}$ & $0.93 \pm 0.34$ & 2.8 \\
\hline Li et al. $(2011 a)^{g}$ & $z<0.05$ & Fixed $=1$ & - & $4.4_{-0.8}^{+0.9} \times 10^{-14}$ & - & 4.4 \\
\hline
\end{tabular}

Notes.

${ }^{\text {a }}$ As derived in Section 6.1 .

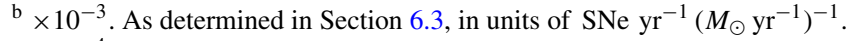

c $\times 10^{-4}$. The estimated $\mathrm{SNR}_{\mathrm{Ia}}$ due to stellar mass, at a fixed stellar mass of $1 \times 10^{10} \mathrm{M}_{\odot}$.

${ }^{\mathrm{d}}$ Results taken from Mannucci et al. (2005) and Scannapieco \& Bildsten (2005).

${ }^{\mathrm{e}}$ Approximating E/S0 galaxies for passive galaxies to determine the value of $A$ and using the rate of SNe Ia in blue $(B-K)$ galaxies to determine the value of $B$.

${ }^{\mathrm{f}}$ Using the global star formation rate as determined by Hopkins \& Beacom (2006).

g Considering elliptical galaxies as passive galaxies.

the rate of SNe Ia as a function of stellar mass in passive galaxies is incompatible with a linear relationship in all cases considered. Having applied the determined offset we find a value of $n_{\mathrm{M}} \sim 0.5$ is preferred, which is consistent with our result at the $1.2 \sigma$ level. The excess rate of SNe Ia in star-forming galaxies is linearly proportional to the SFR in all cases considered. We find some evidence for a lower SNuM in passive galaxies, than determined in Section 6.5, with a maximum difference of $2.2 \sigma$.

\subsection{Comparison to Other Results}

Throughout this analysis we have compared our results to that of Sullivan et al. (2006). We find a different dependence on stellar mass for the $\mathrm{SNR}_{\mathrm{Ia}}$, but agree that there is a strong dependence on the recent SFR. In agreement with the results of Sullivan et al. (2006), we find that the SNR Ia per unit stellar mass is greater in highly star-forming galaxies compared to passive galaxies, with an approximately linear dependence on sSFR except for one potentially anomalous point in the SDSS data. Different assumptions about the dependence of the $\mathrm{SNR}_{\mathrm{Ia}}$ on stellar mass do not significantly alter our conclusions about its dependence on the recent SFR.

A summary of how our results compare to those found by other studies is given in Table 6. Scannapieco \& Bildsten (2005) and Mannucci et al. (2005) also investigated the possibility that the $\mathrm{SNR}_{\mathrm{Ia}}$ may be a two-component model, assuming $n_{\mathrm{M}}=n_{\mathrm{SFR}}=1$. These analyses updated $\mathrm{SN}$ rates from Cappellaro et al. (1999), determining values of $A=3.83_{-1.2}^{+1.4} \times$ $10^{-14} \mathrm{SNe} \mathrm{yr}^{-1} M_{\odot}^{-1}$, for $\mathrm{SNe}$ in $\mathrm{E} / \mathrm{S} 0$ galaxies (which can be crudely associated with passive galaxies in this analysis) and either $B=1_{-0.5}^{+0.6} \times 10^{-3} \mathrm{SNe} \mathrm{yr}^{-1}\left(M_{\odot} \mathrm{yr}^{-1}\right)^{-1}$ or $B=$

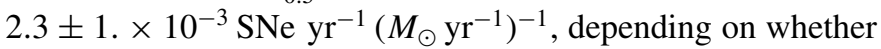
the $z \leqslant 1.0$ core-collapse SN rate density (Dahlen et al. 2004) compared to the SFR density (Giavalisco et al. 2004), or the population of SNe Ia found in blue $(B-K)$ galaxies, is used to model the $\mathrm{SNR}_{\mathrm{Ia}}$ in star-forming galaxies. The value of $A$ is consistent with our result $\left(2.4_{-0.5}^{+0.6} \times 10^{-14} \mathrm{SNe} \mathrm{yr}^{-1} M_{\odot}^{-1}\right)$, when $n_{\mathrm{M}} \equiv 1$ is assumed, and our value of $B=(1.2 \pm 0.1) \times$ $10^{-3} \mathrm{SNe} \mathrm{yr}^{-1}\left(M_{\odot} \mathrm{yr}^{-1}\right)^{-1}$ when $n_{\mathrm{M}} \equiv 1$ and $n_{\mathrm{SFR}} \equiv 1$ is 


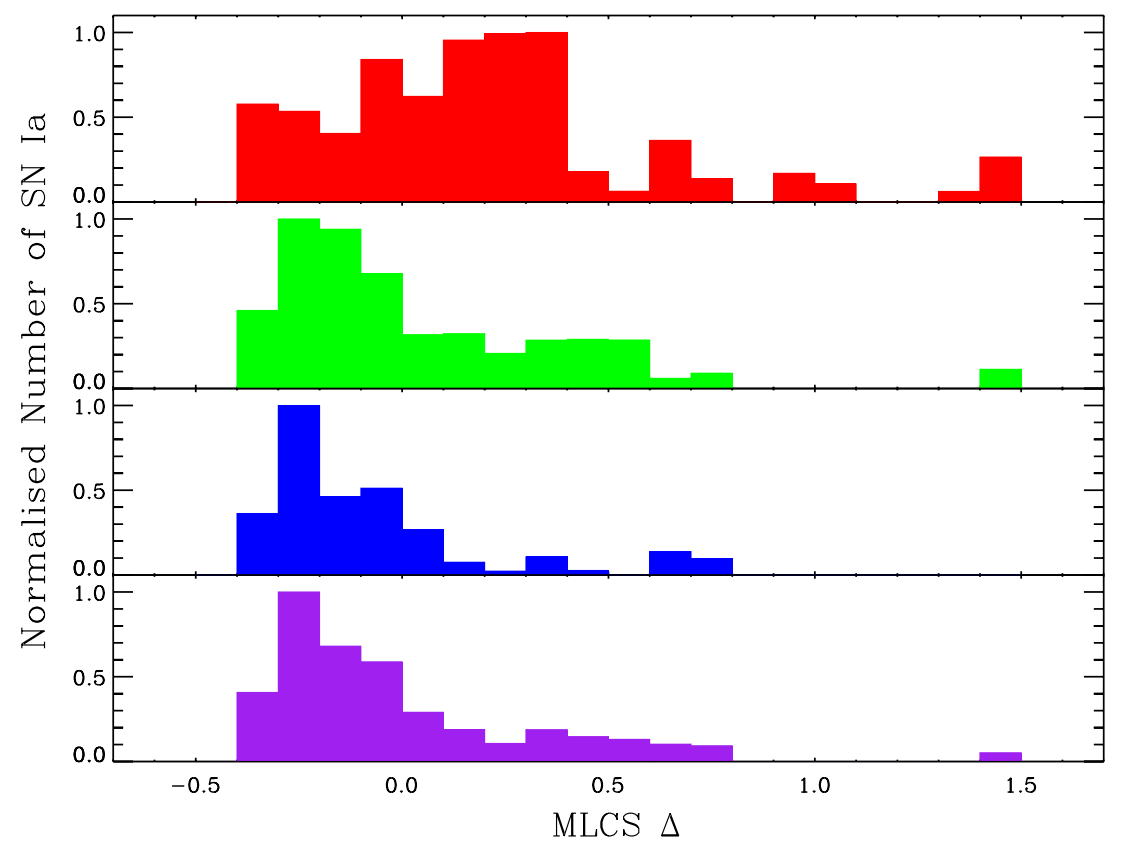

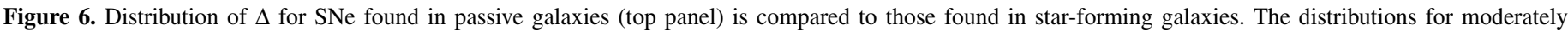

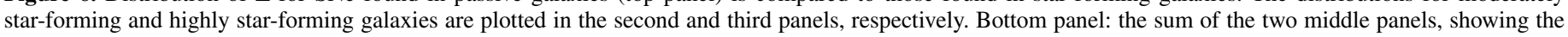
combined distribution for star-forming galaxies.

(A color version of this figure is available in the online journal.)

also in good agreement. However, as noted, the A+B model for the $\mathrm{SNR}_{\mathrm{Ia}}$ that depends linearly on $M$ and SFR does not provide the best fit for our data set. Scannapieco \& Bildsten (2005) and Mannucci et al. (2005) assumed that the SNR $_{\text {Ia }}$ depends linearly on $M$ and SFR. Dilday et al. (2008) using a sample of low-redshift SNe $(z<0.12)$ from the SDSS-II SN Survey (and overlapping with this work) combined with other published work, used the global SFR as determined by Hopkins \& Beacom (2006) (which may overestimate the total mass density) to determine $A=(2.8 \pm 1.2) \times 10^{-14} \mathrm{SNe} \mathrm{yr}^{-1} M_{\odot}^{-1}$ and $B=(0.93 \pm 0.34) \times 10^{-3} \mathrm{SNe} \mathrm{yr}^{-1}\left(M_{\odot} \mathrm{yr}^{-1}\right)^{-1}$, which are in agreement with those found in this analysis. Li et al. (2011a) study how the $\mathrm{SNR}_{\mathrm{Ia}}$ is related to the size, color, and morphology of the host galaxy for a sample of local SNe. They show that the $\mathrm{SNR}_{\mathrm{Ia}}$ is not linearly related to the stellar mass of the host galaxy, instead preferring a relationship, $\mathrm{SNR}_{\mathrm{Ia}} \propto$ $M^{\sim 0.5}$, independent of host galaxy morphology and color. Their result for elliptical galaxies is in excellent agreement with our results for passive galaxies, favoring an $\mathrm{SNR}_{\mathrm{Ia}}$ proportional to $M^{0.72 \pm 0.13}$. However, our results differ for star-forming galaxies, where we find that an $\mathrm{SNR}_{\mathrm{Ia}} \propto M^{1.01 \pm 0.09}$ is favored. Li et al. (2011a) also consider the case where $\mathrm{SNR}_{\mathrm{Ia}} \propto M$ for elliptical galaxies, finding a value of $A=4.4_{-0.8}^{+0.9} \times 10^{-14} \mathrm{SNe} \mathrm{yr}^{-1} M_{\odot}^{-1}$ (in our framework), which is consistent with our result.

Dilday et al. (2010), using a virtually identical sample of $\mathrm{SNe}$ Ia to this analysis, study the evolution of the volumetric $\mathrm{SNe}$ rate. By assuming a model for the evolution of the SFR density as a function of redshift, we can study the consistency of our results. Hopkins \& Beacom (2006), using the commonly used parameterization for the SFR density; $\dot{\rho}=(a+b z) h /\left(1+(z / c)^{d}\right)$, with $h=0.7$ (Cole et al. 2001), find $a=0.0118, b=0.08, c=3.3$, and $d=5.2$, when assuming an IMF from Baldry \& Glazebrook (2003). Evaluating these values for $z=0.2$ (the median redshift of our sample), produces a value of $\dot{\rho}=0.019 M_{\odot} \mathrm{yr}^{-1} \mathrm{Mpc}^{-3}$. Combining this with our determined best-fit value of $B=1.23 \times 10^{-3}$, we

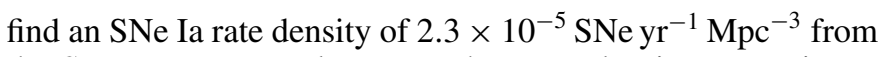
the SFR component alone. For the mass density, we estimate $\log (\rho)=8.5$ from Hopkins \& Beacom (2008), and use the best-fitting value of $A=2.4 \times 10^{-14}$ to estimate a total $\mathrm{SNe}$ Ia rate density of $3.10 \times 10^{-5}$. For comparison, Dilday et al. (2010) find a value of $(3.48 \pm 0.31) \times 10^{-5} \mathrm{SNe} \mathrm{yr}^{-1} \mathrm{Mpc}^{-3}$. For $0.175<z<0.225$, these results are entirely consistent. When $n_{\mathrm{M}}$ is allowed to vary and its uncertainty is considered, the results are also consistent.

\section{SNe PROPERTIES}

We have studied how the rate of SNe Ia is related to the host galaxy properties and have seen that the rate of $\mathrm{SNe}$ is dependent on the galaxy stellar mass and SFR. We extend this analysis to consider how the SN Ia light-curve parameters are related to the host galaxy properties.

Lampeitl et al. (2010b) used a similar sample from the SDSS-II SNe survey to study how SNe Ia observables are related to host galaxy properties and affect their use as cosmological probes. This analysis differs from Lampeitl et al. (2010b) as we have compiled a homogeneously selected sample that is complete to $z<0.25$. We study how the stretch of the SNe Ia, parameterized in MLCS through the $\Delta$ parameter, and the SN color, modeled through the parameter $A_{V}$, are related to the host galaxy properties.

\subsection{MLCS2k2 $\Delta$ Parameter as a Function of Host Galaxy Type}

Figure 6 shows the distribution of the MLCS2k $2 \Delta$ parameter for the $\mathrm{SNe}$ Ia found in passive and star-forming host galaxies (shown both separately and as a combined data set), after correcting for efficiency as described in Sections 3.2 and 4.4.

A Kolmogorov-Smirnov test (K-S test; Chakravarti et al. 1967) is to test the hypothesis that the two histograms shown in Figure 6 are drawn from the same parent distribution. We find a probability of $6.1 \times 10^{-10}$ for the K-S test and conclude 


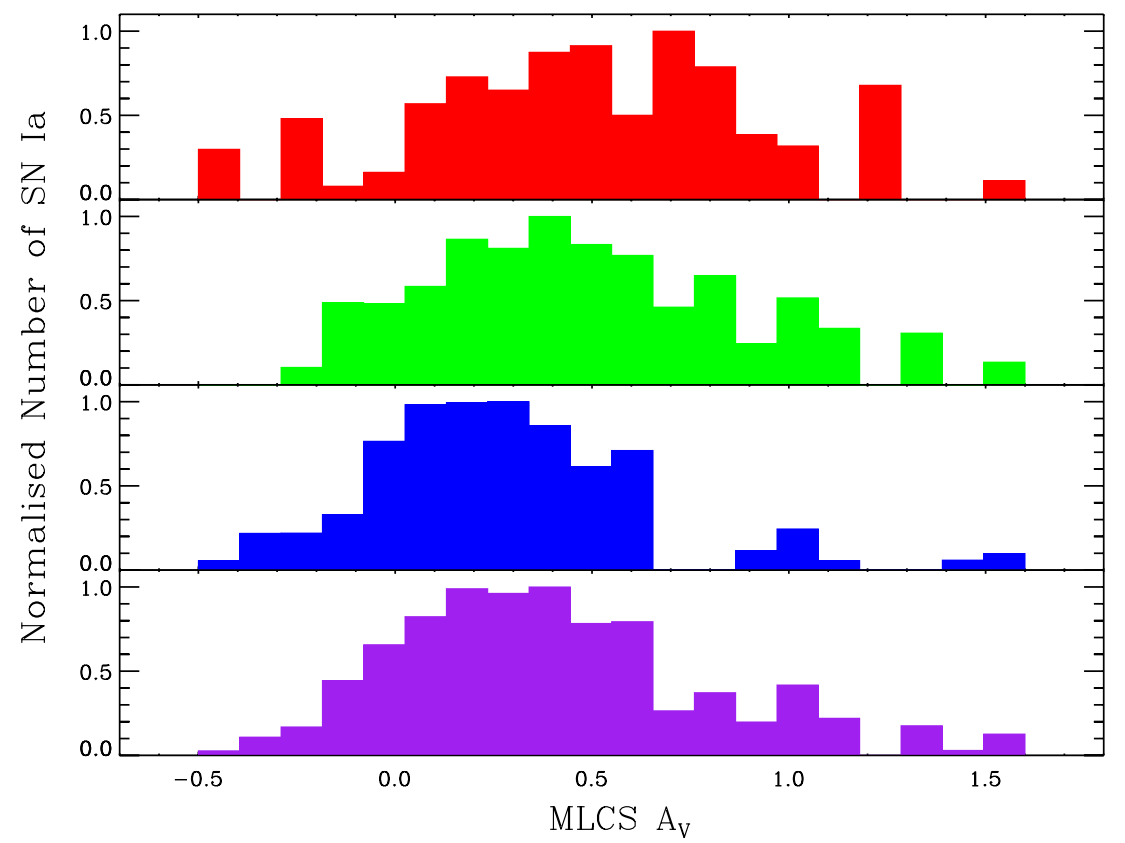

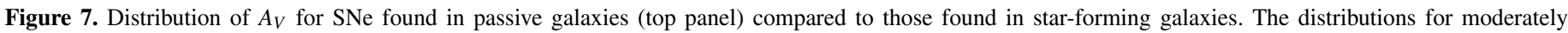

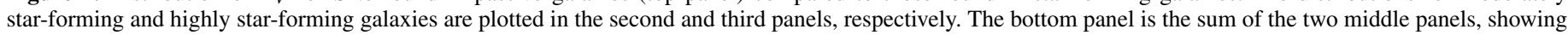
the combined distribution for star-forming galaxies. A flat prior is used in the light-curve fitting.

(A color version of this figure is available in the online journal.)

that the histograms arise from two different populations. We find a K-S test probability of 0.08 that the $\Delta$ distributions in moderately and highly star-forming galaxies arise from the same parent distribution. Our result confirms previous findings (Sullivan et al. 2006, 2010; Lampeitl et al. 2010b) that SNe in star-forming galaxies are brighter than their passive counterparts and that $\mathrm{SNe}$ in passive galaxies exhibit a broader range of $\Delta$ values when compared to their star-forming counterparts.

These results are not surprising in the context of a twocomponent model since we have shown that most of the rate even in moderately star-forming galaxies can be attributed to recent star formation. However, if there were more than two components, or some evolution depending on the starforming rate, we might have observed a significant difference between the highly star-forming and moderately star-forming distributions.

\subsection{MLCS $2 k 2 A_{V}$ Parameter as a Function of Host Galaxy Type}

Determining the color of SNe Ia is important for cosmological parameter estimation. Recently, Sullivan et al. (2010), Kelly et al. (2010), and Lampeitl et al. (2010b) found evidence that $\mathrm{SNe}$ in different environments may follow different color laws. Here we consider how the distribution of color, expressed by the MLCS2k2 $A_{V}$ parameter, varies as a function of host galaxy sSFR. To examine this relationship we apply a flat prior in MLCS2k2, allowing $A_{V}$ to take all values (both positive and negative), so that we are not sensitive to assumptions about the distribution of $A_{V}$ values. The use of a flat prior changes the number of SNe Ia that pass our selection criteria from 342 to 338. The effect of our choice of prior is discussed further in Section 7.3.

Figure 7 shows the distribution of $A_{V}$ for SNe in passive hosts and star-forming galaxies. The distributions for moderately starforming and highly star-forming galaxies are plotted separately, along with the case where the two star-forming data sets have been combined. We use the efficiency correction described in Section 3.2 and the $1 / V_{\max }$ correction determined in Section 4.4 to weight each galaxy. Passive galaxies have a mean $A_{V}$ of 0.40 mag and variance 0.27 , compared to a mean of 0.33 mag and variance 0.15 for star-forming galaxies. For the individual star-forming galaxy populations, we find means of 0.43 and $0.22 \mathrm{mag}$ and variances of 0.16 and 0.12 for moderately and highly star-forming galaxies, respectively.

As in Section 7.1, we use a K-S test to indicate whether the distributions are drawn from the same parent distributions. We find a probability of 0.163 , suggesting no evidence that the distributions may be drawn from different parent distributions. These results are consistent with Lampeitl et al. (2010b), who used the SDSS spectroscopically confirmed SNe fitted with the SALT2 light-curve fitter, and saw no significant difference in the distribution of the SALT2 color parameter, $c$, for SNe Ias in passive and star-forming galaxies.

As before, we split the star-forming data set into moderately and highly star-forming galaxies. We fit K-S test probabilities of 0.42 when passive and moderately star-forming data sets are considered, $8.0 \times 10^{-4}$ for passive and highly star forming, and $2.3 \times 10^{-4}$ between the two star-forming data sets. We conclude that while there is no evidence of a difference in the $A_{V}$ distributions between the passive and moderately star-forming data sets, the highly star-forming sample has a different distribution in $A_{V}$, as shown in Figure 7, with SNe Ia in highly star-forming galaxies on average exhibiting smaller values of $A_{V}$.

Recent studies (e.g., González-Gaitán et al. 2011; Garnavich et al. 2004) have shown that sub-luminous SNe Ia, such as SN 1991bg (Leibundgut et al. 1993), are observed to be redder than normal-type SNe Ia. This sub-type of SNe Ia is typically defined to be those with $s<0.8$, or comparably, $\Delta>0.4$ for MLCS2k2. To ensure that our results are not being driven by these outliers to the $\mathrm{SNe}$ Ia population, we remove candidates from our sample with $\delta>0.4$, and recalculate the K-S test probabilities. We find probabilities of 0.49 when only the 
Table 7

Effect of Our Selection Criteria on the Results Described in Section 7 on the Distribution of $A_{V}$

\begin{tabular}{|c|c|c|c|c|c|}
\hline \multirow[t]{2}{*}{ Selection } & \multirow[t]{2}{*}{ No. of Hosts } & \multicolumn{4}{|c|}{ K-S Test for $A_{V}$ Distribution } \\
\hline & & Passive/Star Forming & Passive/Mod. & Passive/Highs & Mod./Highs \\
\hline Flat $A_{V}$ Prior & 338 & 0.163 & 0.416 & $7.98 \times 10^{-4}$ & $2.31 \times 10^{-4}$ \\
\hline Std $A_{V}$ Prior & 342 & 0.295 & 0.889 & $6.11 \times 10^{-3}$ & $1.10 \times 10^{-4}$ \\
\hline Positive $A_{V}$ Prior & 364 & 0.036 & 0.923 & $3.07 \times 10^{-4}$ & $1.13 \times 10^{-4}$ \\
\hline Confirmed & 197 & 0.320 & 0.843 & 0.022 & $3.13 \times 10^{-3}$ \\
\hline Phot-ID & 145 & 0.573 & 0.390 & 0.271 & 0.018 \\
\hline$z<0.20$ & 196 & 0.542 & 0.606 & 0.034 & $1.00 \times 10^{-4}$ \\
\hline$z<0.16$ & 103 & 0.612 & 0.032 & 0.240 & $8.04 \times 10^{-5}$ \\
\hline
\end{tabular}

passive and moderately star-forming galaxies are considered, $2.3 \times 10^{-4}$ between the passive and highly star-forming data sets and $8.3 \times 10^{-5}$ for moderately and highly star forming. These results are entirely consistent with our fiducial analysis, and show that our results are not due to sub-luminous SNe Ia in our sample.

This analysis assumes that the observed values of $A_{V}$ are good approximations to the true underlying values. However, while the majority of $\mathrm{SNe}$ in our sample have well-measured light curves, resulting in accurate measurements of $A_{V}$, many of the $\mathrm{SNe}$ Ia in our sample have low $\mathrm{S} / \mathrm{N}$ measurements. In such cases, since the underlying distribution of $A_{V}$ for $\mathrm{SNe}$ Ia is observed to be exponentially declining, the measured value of $A_{V}$, for an individual $\mathrm{SNe}$, is more likely to be scattered toward a higher value of $A_{V}$ than a lower value. This would result in a higher proportion of SNe with high $A_{V}$ measurements compared to a distribution of $\mathrm{SNe}$ Ia with high $\mathrm{S} / \mathrm{N}$ light curves.

To rigorously account for $\mathrm{S} / \mathrm{N}$ variations in the observations, the underlying $\Delta$ and $A_{V}$ distributions are determined using the method described in D'Agostini (1995) and Appendix D of Kessler et al. (2009a). To quantify the uncertainty in the underlying distributions, 60 data-sized simulations were analyzed in the same way as the data. The spread in the mean and rms of the extracted distributions are taken to be the uncertainties in these quantities. To avoid pathologies from poorly measured photometric redshifts, only SNe Ia with a spectroscopic redshift (either from the SNe or host galaxy) are used. The resulting incompleteness was modeled in simulations and found to have a negligible impact on the results. Based on the spread in the distribution moments (both mean and rms), we estimate that the distribution of $A_{V}$ in highly star-forming galaxies differs from that of passive and moderately star-forming galaxies at $3.3 \sigma$ and $3.5 \sigma$, respectively.

For our sample, $\mathrm{SNe}$ Ia have similar $\mathrm{S} / \mathrm{N}$ values at maximum brightness, for all host galaxy types. SNe Ia in passive galaxies have a mean $\mathrm{S} / \mathrm{N}$ of 36 (with rms of 26) compared to means of 33 and 42 and variances of 17 and 30 for SNe Ia in moderately star-forming and highly star-forming galaxies, respectively.

Finally, we use the SALT2 light-curve fitter (Guy et al. 2007, 2010) to determine if our results are dependent on light-curvefitting technique. SALT2 uses a color term, $c$, as a measure of the color of an individual SN Ia, but does not explicitly attribute it to dust extinction. We recover the underlying distribution of $c$ for SNe in our sample, following the technique of D'Agostini (1995), and find that the both the mean and rms of the color distribution for SNe Ia in highly star-forming galaxies are different from those in passive and moderately star-forming galaxies at $2.7 \sigma$ and $3.8 \sigma$, respectively.

From Figure 7, we observe that this result is driven by a deficit of the reddest $\mathrm{SNe}$ Ia in highly star-forming galaxies. The distributions of $A_{V}$ are comparable for systems with $A_{V}<0.15$. This appears to differ with the results of Sullivan et al. (2010), who find that SNe Ia in low sSFR galaxies are bluer than the mean. To test this, we consider only SNe Ia with $A_{V} \geqslant 0.15$ and find a K-S test probability of $1.3 \times 10^{-3}$ between passive and highly star-forming galaxies. Conversely, when only $\mathrm{SNe}$ with $A_{V}<0.15$ are considered, we find a K-S test probability of 0.13 , evidence that these two data sets are consistent.

A physical understanding of this difference is unclear. Sargsyan et al. (2010) suggest that there may be an increased amount of dust observed in starburst galaxies. Dust will make $\mathrm{SNe}$ colors redder. However, the reddest SNe Ia are also the faintest $\mathrm{SNe}$ Ia. Passive galaxies may have lower dust levels (Calzetti 2000) than star-forming systems. If the spread in the color distribution of SNe Ia observed in passive galaxies is primarily due to the explosion mechanism, then a population of high $A_{V} \mathrm{SNe}$ Ia may exist in highly star-forming galaxies that are being obscured by the high dust levels of these systems.

However, Salim et al. (2005) argue that the dust content in these systems is smaller, and covers a smaller range, in low stellar mass, highly star-forming galaxies. In this case, if the distribution of $A_{V}$ in passive galaxies is due to the local $\mathrm{SNe}$ environment (Maeda et al. 2011), then we might expect a deficit of the reddest $\mathrm{SNe}$ in strongly star-forming galaxies due to their lower levels of dust.

We are therefore unable to accurately determine the cause of these differences until a better understanding of the dust levels as a function of galaxy sSFR is better understood.

\subsection{The Effect of our Selection Criteria}

In Section 6.6, we considered how the inclusion of nonspectroscopically confirmed $\mathrm{SNe}$ Ia and our redshift range affected the results of Sections 6.1, 6.3, and 6.5. Here, we carry out a similar analysis on the results of Section 7.2. We also consider how the $A_{V}$ prior used in the MLCS2k2 light-curve fits affect our conclusions.

Table 7 shows the K-S test probability described in Section 7.2 for various selection criteria. We consider the "standard" $A_{V}$ prior discussed in Section 3.1, a flat $A_{V}$ prior, as used in Section 7.2, and a prior where $A_{V}$ is forced to positive. We also consider the effect of varying our redshift range, and considering only spectroscopically confirmed SNe Ia.

From Table 7, we see no evidence that the distribution of $A_{V}$ in passive galaxies differs from that seen in star-forming galaxies, matching the result found in Section 7.2. There is evidence that the distribution of $A_{V}$ in highly star-forming galaxies does not match that of passive and moderately star-forming galaxies. We carried out a similar analysis on the distribution of $\Delta$ and found results entirely consistent with those in Section 7.1. 
One of the conclusions of this work is that the distribution of $A_{V}$ for SNe Ia in highly star-forming galaxies differs from that observed in moderately star-forming and passive systems, due to a deficit of the reddest $\mathrm{SNe}$ in these galaxies. From Figure 1, we see that our efficiency is lowest for SNe Ia with $A_{V} \geqslant 0.5$. However, we also observe that the efficiency corrections are comparable for all systems when $z<0.20$. In Table 7 , we test the effect that our redshift limit has on this result. When only SNe Ia with $z<0.20$ are considered, the results are consistent with our fiducial result, indicating that the low efficiency of the high $A_{V} \mathrm{SNe}$ Ia is not responsible for this result.

In Section 7.2, we used the SALT2 light-curve fitter to show that our results concerning the distribution of $A_{V}$ (or $c$ ) for $\mathrm{SNe}$ Ia as a function of host galaxy type are independent of lightcurve-fitting technique considered. SALT2 parameterizes the relationship between the peak luminosity of an SN Ia and the shape of its light curve through the $x_{1}$ parameter. We recover the underlying distribution of $x_{1}$ for $\mathrm{SNe}$ in our sample, and find that both the mean and rms of the $x_{1}$ distribution for $\mathrm{SNe}$ Ia in passive galaxies differ from those found in moderately and highly star-forming galaxies at $4.6 \sigma$ and $9.6 \sigma$, respectively, confirming the results of Section 7.1.

We further considered the possibility that our results may depend on survey conditions or may vary as a function of position on the sky. We split the host galaxy sample by both year and position but saw no significant effects.

\section{CONCLUSIONS}

We have studied how the $\mathrm{SNR}_{\mathrm{Ia}}$ and light-curve properties depend on the host galaxy stellar mass and SFR. By augmenting the SDSS spectroscopically confirmed SNe Ia with SNe identified by only their light curves, we have constructed a large, homogeneous, and well-understood sample of $342 \mathrm{SNe}$ Ia in the redshift range $0.05<z<0.25$. Our sample has low contamination and is unbiased with respect to spectroscopic selection effects and survey conditions. The efficiency of the SDSS-II SN Survey is well measured in this redshift range, allowing us to study the overall $\mathrm{SNR}_{\mathrm{Ia}}$ as a function of these host galaxy properties. We summarize below the main conclusions of this work.

1. We find that the $\mathrm{SNR}_{\mathrm{Ia}}$ in passive galaxies is not linearly proportional to the stellar mass, but instead favoring $\mathrm{SNR}_{\mathrm{Ia}} \propto M^{0.72}$, as illustrated in Figure 3. This result differs from that of Sullivan et al. (2006), at higher redshift, who favor a linear relationship, but is in good agreement with the conclusions of Li et al. (2011a), who favor $\mathrm{SNR}_{\mathrm{Ia}} \propto M^{0.487 \pm 0.316}$ for elliptical galaxies in the local universe.

2. We show that our results, for $\mathrm{SNe}$ Ia in passive galaxies, are consistent with those of Sullivan et al. (2006) when we account for the difference in the ages of our galaxies. Galaxies at a fixed stellar mass will be older at $z=0.25$ compared to $z=0.75$. When only considering galaxies with $M_{\text {stellar }}<11.3$, we recover a slope consistent with a linear relationship between the $\mathrm{SNR}_{\mathrm{Ia}}$ and $M_{\text {stellar }}$. This is evidence that the rate of SNe Ia is correlated with the age of the stellar population, $\mathrm{SNR}_{\mathrm{Ia}} \propto t^{-1}$, with the most massive galaxies having a deficit in the number of SNe Ia compared to their lower mass counterparts, due to the older stellar population in these galaxies.

3. For star-forming galaxies, we find that the $\mathrm{SNR}_{\mathrm{Ia}}$ as a function stellar mass differs from that of passive galaxies, instead favoring $\mathrm{SNR}_{\mathrm{Ia}} \propto M^{1.01}$. This result differs from that of Li et al. (2011a), who found that the $\mathrm{SNR}_{\mathrm{Ia}}$ as a function of stellar mass is independent of host galaxy morphology and color.

4. We show that the $\mathrm{SNR}_{\mathrm{Ia}}$ per unit stellar mass is a function of sSFR, with SNe Ia being preferentially found in highly star-forming or starburst galaxies, compared to their passive counterparts (Figure 5). Excluding one systematically uncertain measurement, this result is consistent with those found by Mannucci et al. (2005) and Sullivan et al. (2006), locally and at high redshift, respectively, implying that $\mathrm{SNe}$ Ia are preferentially found in highly star-forming galaxies at all redshifts.

5. We demonstrate that the excess $\mathrm{SNR}_{\mathrm{Ia}}$ in star-forming galaxies is well fitted by a linear relationship proportional to the recent SFR, as shown in Figure 4. The component related to recent star formation is the dominant contributor to the $\mathrm{SNR}_{\mathrm{Ia}}$ in these galaxies.

6. We find that a bivariate fitting technique confirms that $\mathrm{SNe}$ Ia in this sample satisfy an $\mathrm{SNR}_{\mathrm{Ia}}$ of the form

$$
\begin{aligned}
\mathrm{SNR}_{\mathrm{Ia}}= & (0.41 \pm 0.15) \times 10^{-10} M^{0.72 \pm 0.15} \\
& +(0.65 \pm 0.25) \times 10^{-3} \mathrm{SFR}^{1.01 \pm 0.22}
\end{aligned}
$$

(statistical errors only). This parameterization is a generalization of the $\mathrm{A}+\mathrm{B}$ model and provides a better fit to the SDSS-II SN data than assuming an $\mathrm{SNR}_{\mathrm{Ia}}$ linearly dependent on stellar mass and SFR.

7. We have tested the effect of our selection criteria on these results, and find that variations in redshift range do not significantly alter our results.

8. We see no difference in the distribution of the extinction parameter, $A_{V}$, between passive and star-forming galaxies as illustrated in Figure 7 . We find no evidence that the distribution of $A_{V}$ in passive galaxies differs from that of moderately star-forming galaxies, but find evidence that the distribution is different for highly star-forming galaxies, which favor lower mean values of $A_{V}$. This result appears to be driven by a deficit of the reddest $\left(A_{V}>0.15\right) \mathrm{SNe}$ Ia in highly star-forming galaxies. This differs from the result of Sullivan et al. (2010), who find an excess of the bluest SNe in low sSFR galaxies. We theorize that this may be due to the high levels of dust in these systems, obscuring the reddest and faintest $\mathrm{SNe}$ Ia. We remove sub-luminous $\mathrm{SNe}$ Ia from our analysis and find that our results are unaffected. We use the method described in Appendix D of Kessler et al. (2009a) to determine the underlying distribution of $A_{V}$ for various galaxy types, and show that the distribution of $A_{V}$ in highly star-forming galaxies differs at the $3 \sigma$ level from that of passive and moderately star-forming galaxies. We find that the choice of $A_{V}$ prior used in the light-curve fitting does not affect our conclusions. We find the same results using the SALT2 model to extract the distribution of color, $c$, thus providing evidence that the difference in the distribution of $A_{V}$ (or $c$ ) is a model-independent feature.

9. We perform a rigorous test of the PÉGASE. 2 SEDs and the Z-PEG fitting technique and find a systematic offset in the photometric redshift estimates produced for our comparison field sample. If a simple correction is applied to both the redshifts and stellar masses of our field sample, we find that our conclusions are unchanged.

The process used to determine our sample of host galaxies and their derived properties allows us to directly compare our conclusions with those of Sullivan et al. (2006), who studied 
the $\mathrm{SNR}_{\mathrm{Ia}}$ at higher redshifts. Sullivan et al. (2006) found the same trends with SFR, but with a different relationship parameterizing the stellar mass into the $\mathrm{SNR}_{\mathrm{Ia}}$. It is unlikely that these differences are due to an evolution of the galaxy population but may reflect that $\mathrm{SNe}$ Ia are primarily triggered by recent bursts of star formation in a galaxy, causing uncertainties in the contribution due to the stellar mass.

We thank the anonymous referee for a detailed reading of this manuscript. The authors thank Mark Sullivan, Damien Le Borgne, Claudia Maraston, and Janine Pforr for helpful discussions. M.S. is funded by an SKA fellowship, while R.C.N. and H.L. are supported by STFC. M.S. thanks Prina Patel and Russell Johnston for insightful comments. Support for this research at Rutgers University was provided in part by NSF CAREER award AST-0847157 to S.W.J.

Funding for the SDSS and SDSS-II has been provided by the Alfred P. Sloan Foundation, the Participating Institutions, the National Science Foundation, the U.S. Department of Energy, the National Aeronautics and Space Administration, the Japanese Monbukagakusho, the Max Planck Society, and the Higher Education Funding Council for England. The SDSS Web site is http://www.sdss.org/.

The SDSS is managed by the Astrophysical Research Consortium for the Participating Institutions. The Participating Institutions are the American Museum of Natural History, Astrophysical Institute Potsdam, University of Basel, University of Cambridge, Case Western Reserve University, University of Chicago, Drexel University, Fermilab, the Institute for Advanced Study, the Japan Participation Group, Johns Hopkins University, the Joint Institute for Nuclear Astrophysics, the Kavli Institute for Particle Astrophysics and Cosmology, the Korean Scientist Group, the Chinese Academy of Sciences (LAMOST), Los Alamos National Laboratory, the Max-PlanckInstitute for Astronomy (MPIA), the Max-Planck-Institute for Astrophysics (MPA), New Mexico State University, Ohio State University, University of Pittsburgh, University of Portsmouth, Princeton University, the United States Naval Observatory, and the University of Washington.

This work is based in part on observations made at the following telescopes. The Hobby-Eberly Telescope (HET) is a joint project of the University of Texas at Austin, the Pennsylvania State University, Stanford University, Ludwig-MaximilliansUniversität München, and Georg-August-Universität Göttingen. The HET is named in honor of its principal benefactors, William P. Hobby and Robert E. Eberly. The Marcario Low-Resolution Spectrograph is named for Mike Marcario of High Lonesome Optics, who fabricated several optical elements for the instrument but died before its completion; it is a joint project of the Hobby-Eberly Telescope partnership and the Instituto de Astronomía de la Universidad Nacional Autónoma de México. The Apache Point Observatory $3.5 \mathrm{~m}$ telescope is owned and operated by the Astrophysical Research Consortium. We thank the observatory director, Suzanne Hawley, and site manager, Bruce Gillespie, for their support of this project. The Subaru Telescope is operated by the National Astronomical Observatory of Japan. The William Herschel Telescope is operated by the Isaac Newton Group, on the island of La Palma in the Spanish Observatorio del Roque de los Muchachos of the Instituto de Astrofisica de Canarias. Based on observations made with the Nordic Optical Telescope, operated on the island of La Palma jointly by Denmark, Finland, Iceland, Norway, and Sweden, in the Spanish Observatorio del Roque de los Muchachos of the

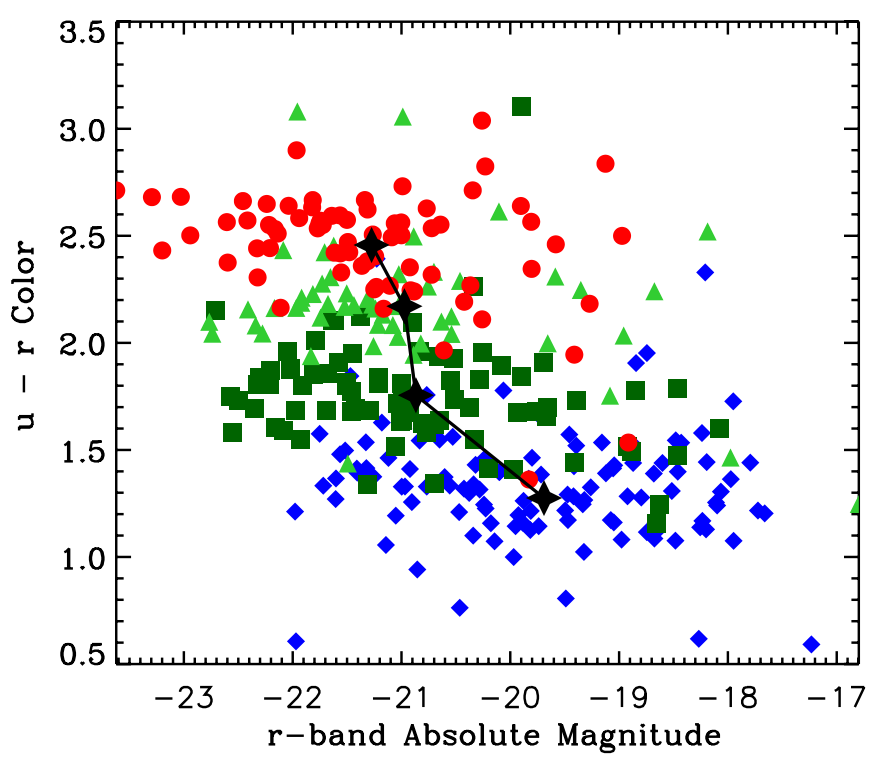

Figure 8. Color $(u-r)$ vs. absolute magnitude for the host galaxy sample used in this analysis. Galaxies classified as passive are plotted as red circles, with highly star-forming galaxies shown as blue diamonds. Moderately star-forming galaxies are plotted in green, with light green triangles indicating the "ridge line" of galaxies discussed in Section 5 and shown in Figure 2. The mean of each individual distribution is shown as a black star to indicate the relationship between color and brightness for the various samples.

(A color version of this figure is available in the online journal.)

Instituto de Astrofisica de Canarias. Kitt Peak National Observatory, National Optical Astronomy Observatory, is operated by the Association of Universities for Research in Astronomy, Inc. (AURA) under cooperative agreement with the National Science Foundation. The W. M. Keck Observatory is operated as a scientific partnership among the California Institute of Technology, the University of California, and the National Aeronautics and Space Administration. The observatory was made possible by the generous financial support of the W. M. Keck Foundation. Based partially on observations made with the Italian Telescopio Nazionale Galileo (TNG) operated on the island of La Palma by the Fundación Galileo Galilei of the INAF (Istituto Nazionale di Astrofisica) at the Spanish Observatorio del Roque de los Muchachos of the Instituto de Astrofísica de Canarias.

\section{APPENDIX A}

\section{COLOR-MAGNITUDE DIAGRAM}

In Section 5, we considered how our host galaxies are distributed as a function of stellar mass and SFR, and noted the presence of a subset of galaxies with low levels of sSFR. These galaxies do not exhibit high $\chi^{2}$ values. Figure 8 shows the color-magnitude diagram for the host galaxies used in this analysis. The sample is split into passive, moderately, and highly star forming as described in the caption for Figure 2. The subset of 55 galaxies with low levels of sSFR $(-11.0<$ sSFR $<10.5)$ are plotted as light green triangles. We see that the PÉGASE.2 SED primarily determine the level of star formation activity in a galaxy based on its color, with passive galaxies being the "reddest" and brightest galaxies through to the "bluest" galaxies being classified as highly star forming. From Figure 8, the population of objects with low sSFR but classified as moderately star forming is observed to lie between the passive and the other moderately star-forming galaxies in color-magnitude space, making their classification 

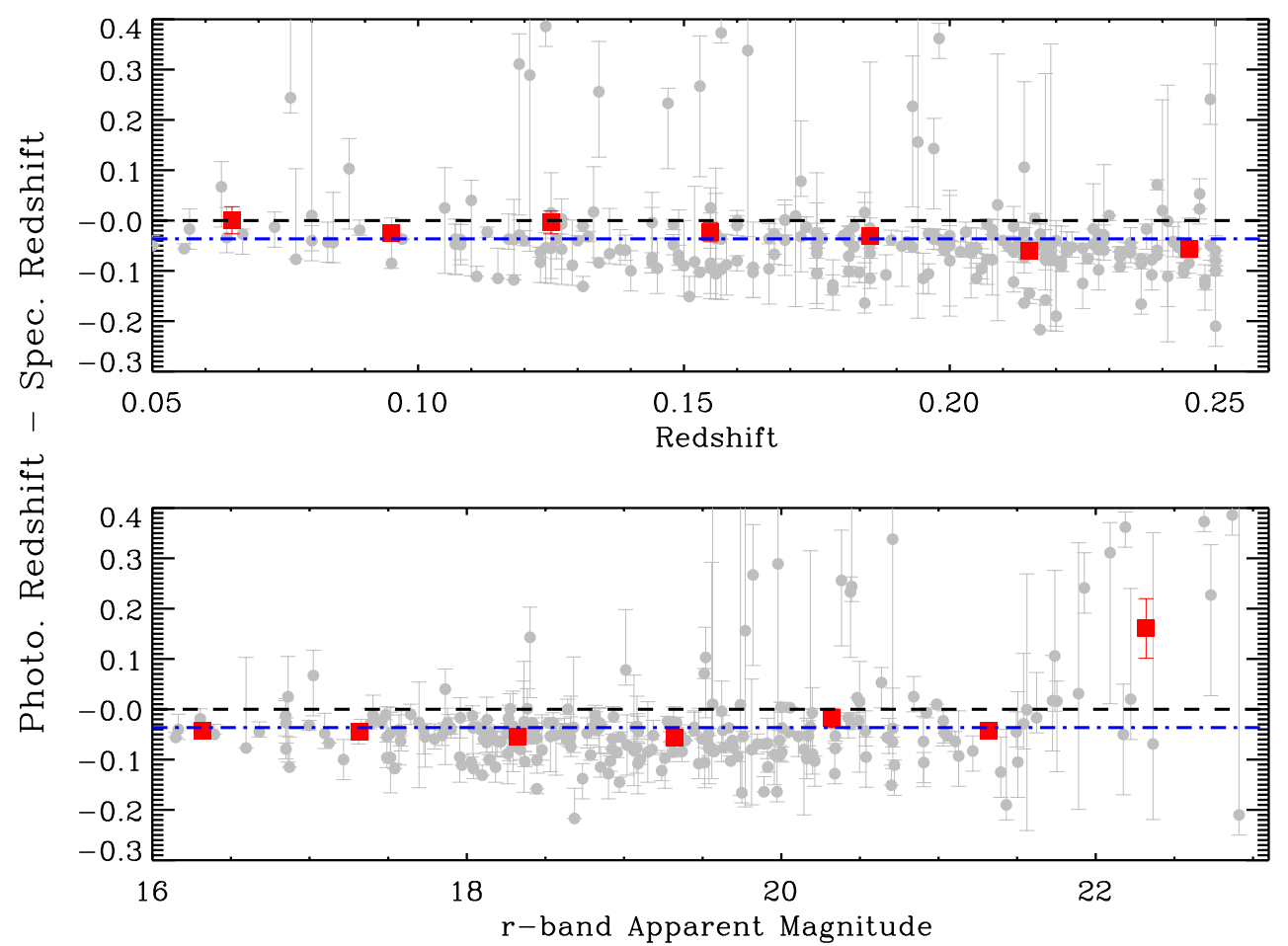

Figure 9. Top: the difference between the photometric redshift estimates derived from the PÉGASE.2 SEDs and the spectroscopic redshift as a function of redshift. Bottom: same as above, as a function of host galaxy apparent magnitude. Individual galaxies are plotted in gray, with red points indicating the values determined when the sample has been binned. The black, dashed line indicates no difference, while the blue (dash-dotted) line indicates the mean difference.

(A color version of this figure is available in the online journal.)

understandable, if ambiguous. Figure 8 uses absolute magnitudes and colors, but the same conclusions can be drawn when apparent magnitudes are considered. We note that, 43 of the 55 (78\%) "ridge-line" galaxies are best described by the lenticular (S0) scenario, with the remaining 12 being best fitted by the elliptical galaxy template, possibly highlighting the uncertainty in the nature of lenticular galaxies. In comparison, only 41 of the $79(52 \%)$ remaining moderately star-forming galaxies are best fitted by an S0 template.

To further investigate the nature of the "ridge-line" galaxies, we study how the distribution of $\Delta$ for $\mathrm{SNe}$ in these galaxies compare to those in moderately star-forming and passive galaxies, since, as described in Section 7.2, there is a significant difference in the $\Delta$ distributions for SN Ia occurring in these galaxies. A K-S test between the distribution of $\Delta$ in passive galaxies to that of "ridge-line galaxies" yields a probability of $1.9 \times 10^{-5}$ that they are drawn from the same parent distribution. This compares to a probability of 0.28 between the distribution of $\Delta$ in "ridge-line" galaxies and other moderately star-forming galaxies. These results further strengthen the conclusion that the "ridge-line" galaxies are not misclassified passive galaxies.

We thus determine that this population of objects is well defined according to color-magnitude space, and that the PÉGASE.2 SEDs use this information to determine the level of star formation activity in each host galaxy.

\section{APPENDIX B}

\section{THE PÉGASE.2 PHOTOMETRIC REDSHIFTS}

One of the key systematic uncertainties in this analysis concerns the accuracy of the derived properties of the comparison field sample used, and in particular the photometric redshift estimates produced by the PÉGASE.2 SEDs. These redshift es- timates will affect not only the number of field galaxies, but also their associated stellar masses. The photometric redshift estimates have been tested at high redshift by Sullivan et al. (2006), but have not been extensively used in the local universe.

To test the accuracy of the photometric redshifts, we use the host galaxy sample described in Section 4, and whose properties are listed in Table 3 . This sample covers the magnitude range of the comparison field sample and is large enough to statistically determine if the photometric estimates are accurate. Figure 9 shows the difference between the photometric redshift estimates and the known spectroscopic redshift for this sample as a function of both redshift and apparent magnitude. We find a mean difference of 0.03 in redshift, with the photometric redshifts being smaller than the spectroscopic redshift. From Figure 9, there is no evidence of this offset being dependent on either the redshift or apparent magnitude of the host galaxy, although the scatter does increase with apparent magnitude.

This observed offset in the photometric redshift will also lead to an incorrect value for the galaxy's stellar mass. To quantify this, we consider the derived stellar mass when the redshift is held fixed, compared to that when it is allowed to float in Figure 10, resulting in the offset described above. An offset of $\log M=0.22$ is seen, with the stellar masses derived when the redshift is allowed to float being smaller than the value determined when the redshift is known.

\section{APPENDIX C}

\section{THE EFFECT OF THE OBSERVED OFFSET ON THE CONCLUSIONS OF THIS WORK}

The offset between the photometric redshifts produced by the PÉGASE.2 SEDs and the spectroscopic redshifts for the host 


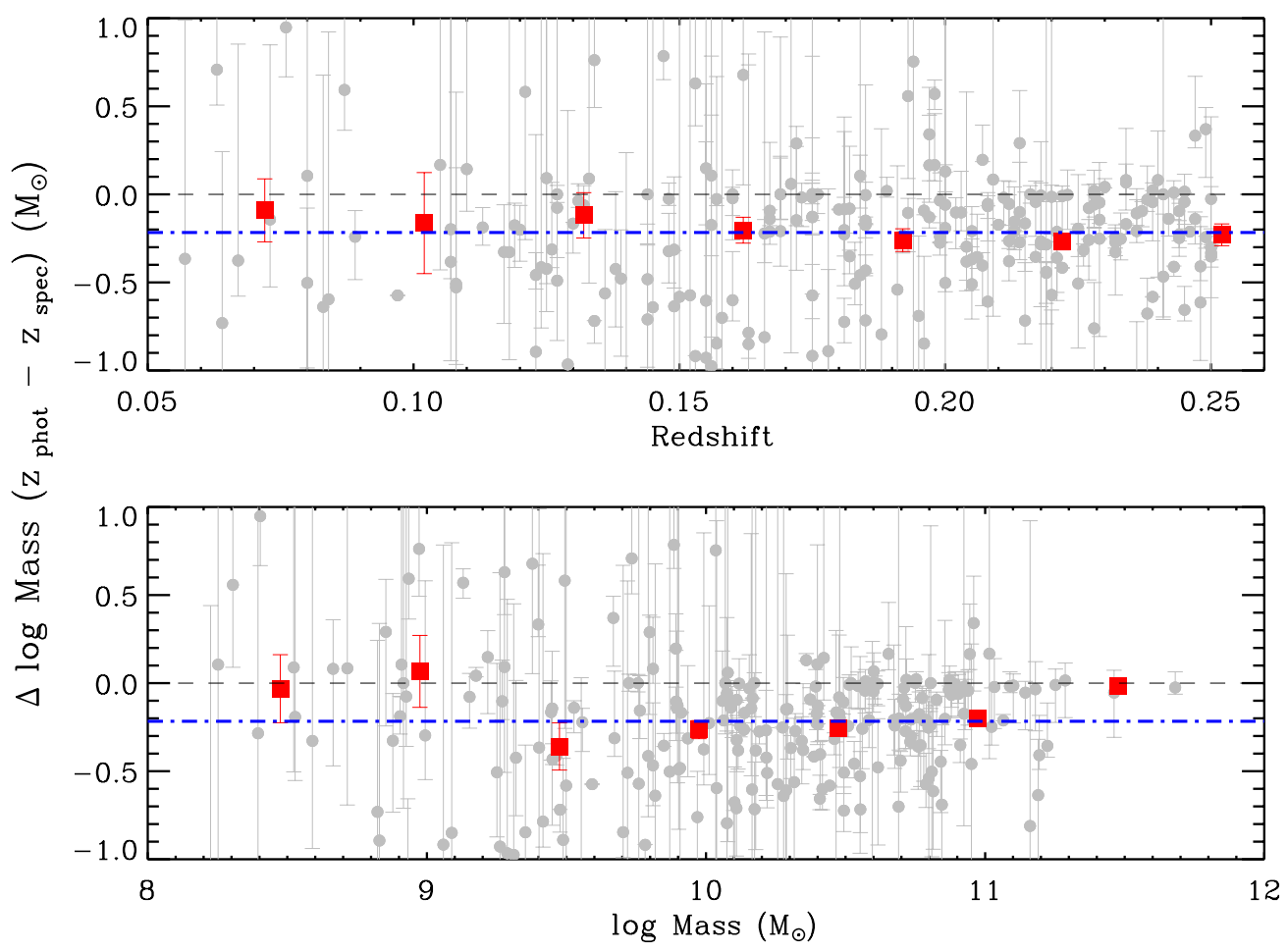

Figure 10. Top: the difference between the stellar mass derived when the redshift is allowed to float in the Z-PEG code compared to that when the redshift is held fixed, resulting in the offset described in Figure 9, as a function of redshift. Bottom: same as above, as a function of stellar mass, as derived when the redshift is held fixed. Individual galaxies are plotted in gray, with red points indicating the values determined when the sample has been binned. The black, dashed line indicates no difference, while the blue (dash-dotted) line indicates the mean difference.

(A color version of this figure is available in the online journal.)

galaxy sample implies that the distribution of galaxies in the comparison field sample used in this analysis does not accurately reflect the distribution of galaxies in our redshift range. This may affect the results of Section 6 . Here we attempt to quantify this systematic uncertainty.

In Appendix A, we observed that there is a strong dependence between the color of the host galaxy and the best-fitting PÉGASE. 2 template determined by the Z-PEG code. This is true for colors determined both by using absolute and apparent magnitudes. The reddest galaxies (in $u-r$ ) are well fitted by a passive template, through to the bluest galaxies, which are considered to be highly star forming. Thus, it appears that we can approximately describe the level of star formation inferred by the PÉGASE. 2 templates as purely a function of observed quantities, and not affected by the offset described in Appendix B. We hence assume, for this analysis, that the offset in the photometric redshifts determined from the PÉGASE.2 SEDs in our redshift range purely affect the inferred stellar masses and not the SFRs. We note that this approximation will only be valid for galaxies spanning a narrow redshift range, as large relative $k$-correction terms can lead to a color dependence, affecting the relationship determined in Figure 8.

In Appendix B, we showed that there is no evidence that the difference in redshift $\left(\left|z_{\text {photo }}-z_{\text {spec }}\right|\right)$ and difference in stellar mass $\left(\left|\log M\left(z_{\text {photo }}\right)-\log M\left(z_{\text {spec }}\right)\right|\right)$ are dependent on either redshift or apparent magnitude. We thus assume that the photometric redshifts derived from the PÉGASE.2 SEDs and associated stellar masses can be offset by the values determined in Appendix B. Table 8 shows the effect that correcting the redshifts and stellar masses of the comparison field sample has on several of the key parameters discussed in this work, when the differences found in Appendix B are applied.
Table 8

Table Showing How the SN Rate Parameters Determined in This Paper Are Altered When the Offsets in Redshift and Stellar Mass, as Determined in Appendix B, Are Applied to the Comparison Field Sample

\begin{tabular}{lcc}
\hline \hline Parameter & Original Result & Mean Offset \\
\hline No. of field galaxies $^{\mathrm{a}}$ & 733688 & 615906 \\
Total stellar mass of field galaxies $^{\mathrm{a}, \mathrm{b}}$ & 6.67 & 9.50 \\
Passive rate $^{\mathrm{c}}$ & $3.56 \pm 0.45$ & $2.49 \pm 0.31$ \\
$n_{\mathrm{M}}{ }^{\mathrm{d}}$ & $0.680 \pm 0.150$ & $0.445 \pm 0.120$ \\
$n_{\mathrm{SFR}}{ }^{\mathrm{e}}$ & $0.940 \pm 0.078$ & $0.782 \pm 0.061$ \\
$n_{\mathrm{SFR}}\left(\text { when } n_{\mathrm{M}}=1\right)^{\mathrm{f}}$ & $0.987 \pm 0.081$ & $1.070 \pm 0.075$ \\
$n_{\mathrm{SFR}}\left(\text { when } n_{\mathrm{M}} \neq 1\right)^{\mathrm{g}}$ & $0.955 \pm 0.074$ & $1.041 \pm 0.070$ \\
\hline
\end{tabular}

Notes.

${ }^{a}$ After the magnitude cut $(15.5<r<23.0)$ and redshift cut $(0.05<z<0.25)$.

${ }^{\mathrm{b}}$ In units of $1 \times 10^{15} M_{\odot}$.

${ }^{c}$ The $\mathrm{SN}$ rate per unit stellar mass per year in passive galaxies, as described in Section 6.5 , in units of $1 \times 10^{-14}$ per unit stellar mass per year.

${ }^{\mathrm{d}}$ The $\mathrm{SN}$ rate per galaxy per year for passive galaxies as a function of log stellar mass, as described in Section 6.1.

${ }^{\mathrm{e}}$ As $d$, except for all star-forming galaxies combined, as described in Section 6.1.

${ }^{\mathrm{f}}$ The SN rate per galaxy per year for star-forming galaxies as a function of log star formation rate, after assuming a component proportional to the stellar mass, as described in Section 6.3.

g As $f$, only assuming a component proportional to the values determined in $d$, as described in Section 6.3.

From Table 8 , it is clear that the number of field galaxies in the redshift range, $0.05<z<0.25$, is dramatically reduced when this corrections is applied, but there is also an increase in stellar mass of each galaxy, resulting in the total stellar mass of the field sample being increased from when no correction is applied. Consequently, the $\mathrm{SNR}_{\mathrm{Ia}}$ per unit stellar mass per 

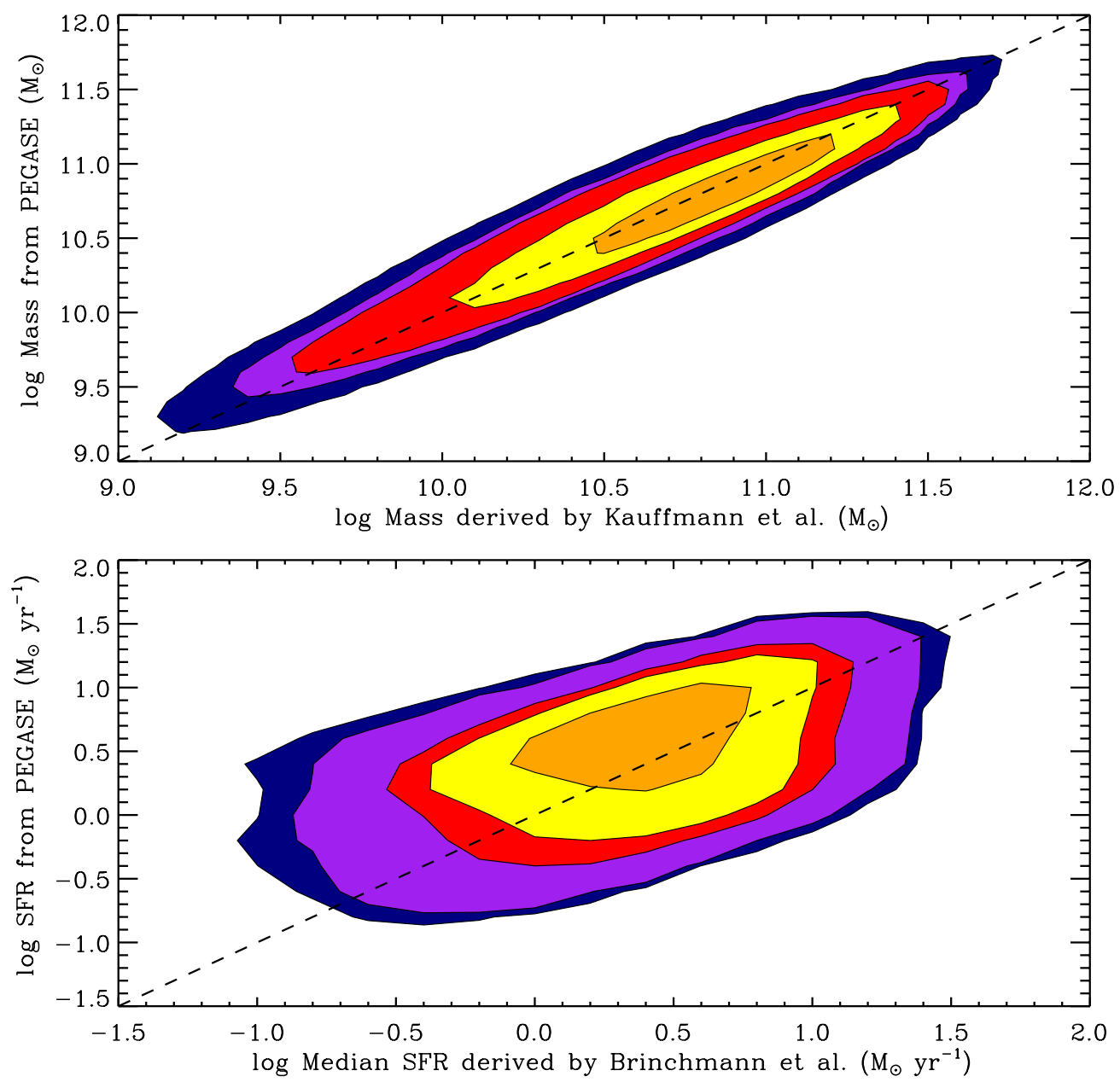

Figure 11. Top: log stellar mass derived from the PÉGASE. 2 templates for a sample of $\sim 330,000$ galaxies from SDSS-I, compared to the estimates obtained from spectral features by Kauffmann et al. (2003). Bottom: same as above, comparing PÉGASE.2 star formation rates to those of Brinchmann et al. (2004). Contours enclose $99 \%$ (dark blue), $95 \%$ (purple), $90 \%$ (red), 68\% (yellow), and 35\% (orange) of the data, respectively.

(A color version of this figure is available in the online journal.)

year in passive galaxies is decreased (at the $2.4 \sigma$ level, when only statistical errors are considered) when the correction is made. This is still in good agreement with other measurements (Sullivan et al. 2006; Mannucci et al. 2005).

When considering the effect that the offset in redshift and stellar mass has on the exponents considered for the $\mathrm{SNR}_{\mathrm{Ia}}$, we see that the value of the slope determined in Section 6.1 decreases, although only by $1.6 \sigma$. In both cases, a linear relationship between the $\mathrm{SNR}_{\mathrm{Ia}}$ per galaxy per year and stellar mass in passive galaxies is strongly disfavored, confirming the results of Section 6.1.

There is a corresponding decrease in the relationship between $\mathrm{SNR}_{\mathrm{Ia}}$ and stellar mass for star-forming galaxies (at the $2.0 \sigma$ level), although as in the main result, there is a clear difference between the rate in passive galaxies when compared to that in star-forming galaxies, indicating the need for an $\mathrm{SNR}_{\mathrm{Ia}}$ that is dependent on more than stellar mass. Finally, we consider the results of Section 6.3. These results are statistically unaffected by the offset discovered in Appendix B. We thus conclude that the main results of this work, namely, that the SN Ia in passive galaxies is not linearly related to the stellar mass, and that the $\mathrm{SNR}_{\mathrm{Ia}}$ in star-forming galaxies is dominated by any recent burst of star formation, are not dependent on issues surrounding the ability of PÉGASE. 2 to accurately determine the photometric redshifts for our comparison field sample. These corrections are not applied in our analysis as a clear understanding of the cause of this offset has not been found, and thus we have only been able to estimate the magnitude of its effect on our results.

\section{APPENDIX D}

\section{THE PÉGASE.2 STELLAR MASS AND SFR ESTIMATES}

In Appendix B, we compared the PÉGASE. 2 photometric redshifts to a similarly distributed sample of galaxies with spectroscopic redshifts, and determined a bias in both redshift and stellar mass. Here, we consider how our derived properties from the PÉGASE. 2 SEDs compare to those determined from the spectral features of a sample of SDSS-I galaxies. Kauffmann et al. (2003) and Brinchmann et al. (2004) used the $4000 \AA$ break and the Balmer absorption line index $\left(H \delta_{A}\right)$ to measure the stellar masses and instantaneous SFRs for galaxies in the SDSS-I DR4 spectroscopic catalog (Adelman-McCarthy et al. 2006).

While a comparison between the stellar mass and SFRs determined by the PÉGASE. 2 templates and the results of Kauffmann et al. (2003) and Brinchmann et al. (2004) may have limitations (this sample consists of only the brightest galaxies in our host galaxy sample, the resolution of the SDSS-I 


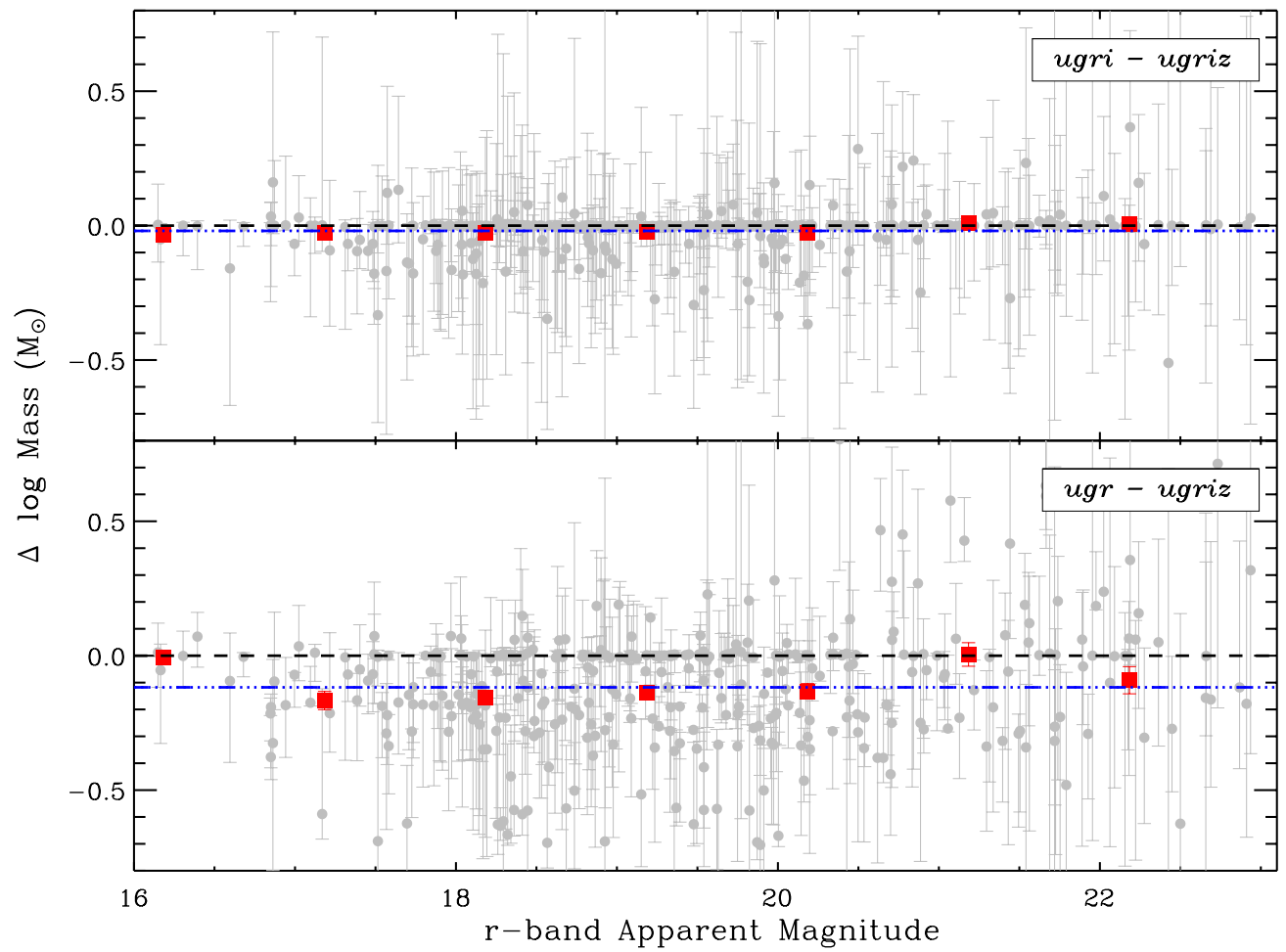

Figure 12. Top: the stellar mass derived from the PÉGASE.2 SEDs when all five SDSS filters ugriz are used in fit compared to when only ugri are used as a function of apparent magnitude. Bottom: same as above, except only three filters (ugr) are used. Individual galaxies are plotted in gray, with red squares indicating the values determined when the sample has been binned. The black, dashed line indicates no difference, while the blue (dash-dotted) line indicates the mean difference.

(A color version of this figure is available in the online journal.)

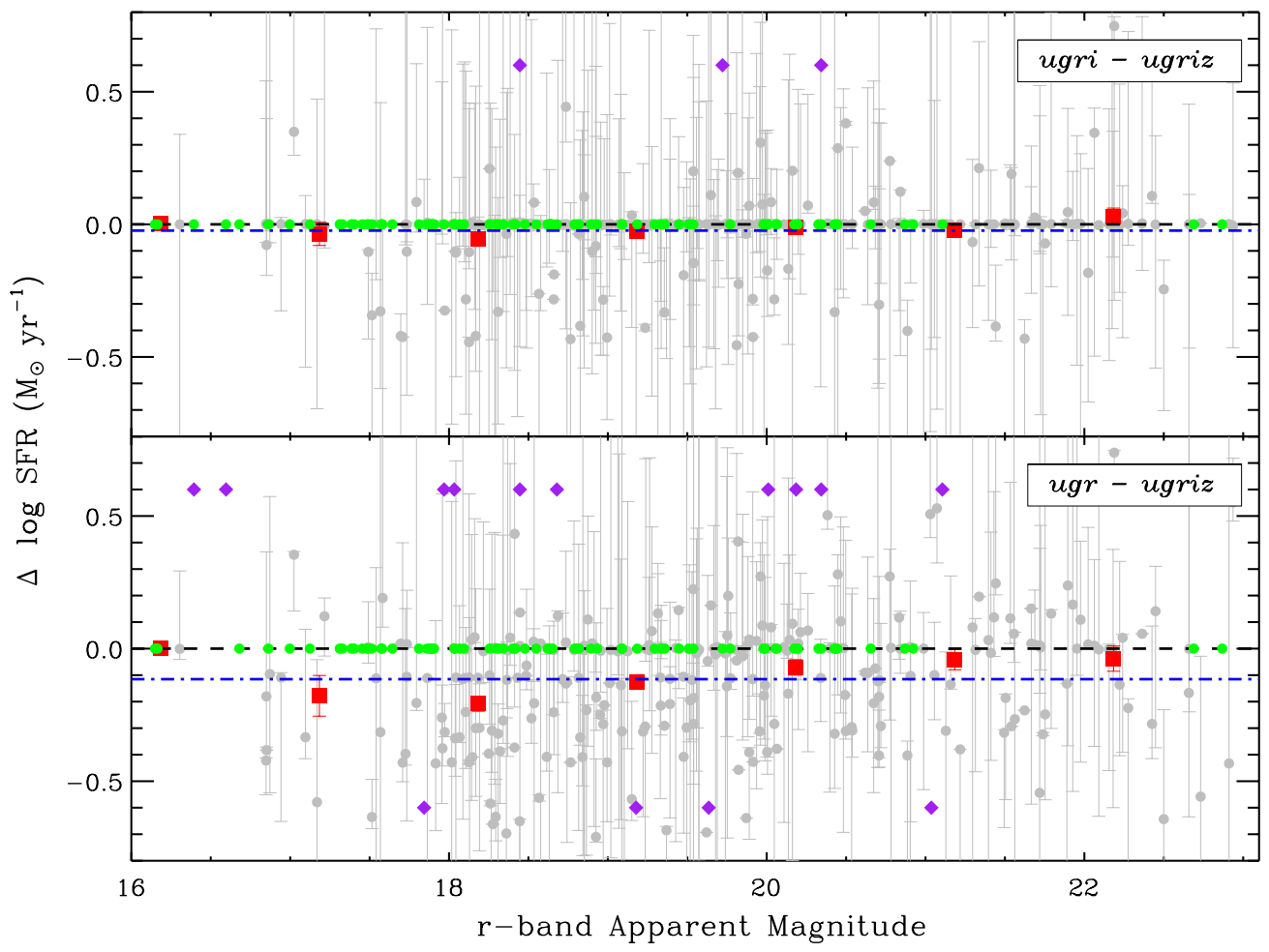

Figure 13. Top: the star formation rate derived from the PÉGASE.2 SEDs when all five SDSS filters ugriz are used in fit compared to when only ugri are used as a function of apparent magnitude. Bottom: same as above, except only three filters (ugr) are used. Individual star-forming galaxies are plotted in gray, with red points indicating the values determined when these galaxies have been binned. Green points (plotted at $\Delta \log (\mathrm{SFR})=0)$ indicate those that are determined to be passive in both cases, while purple diamonds (shown here at \pm 0.6 ) are those which are determined to be star forming in only one scenario. The black, dashed line indicates no difference, while the blue (dash-dotted) line indicates the mean difference.

(A color version of this figure is available in the online journal.) 
spectra is not optimal, and this method relies on the same underlying physics as the PÉGASE. 2 templates), it provides a useful validation of the PÉGASE. 2 measurements. We use $\sim 330,000$ galaxies from the SDSS-I catalog, limiting ourselves to the redshift range considered in this analysis. Several spectral measurements are available; we use the dust-corrected stellar mass (median value) and total SFR (median of the likelihood distribution), and determine estimates from the PÉGASE. 2 SEDs using the spectroscopic redshifts and model magnitudes.

Figure 11 shows the relationship between the spectroscopic and photometric estimates of stellar mass and SFR. The total stellar mass is well recovered, with a mean offset of only $\Delta \log M=0.001$, and variance 0.028 , well below the range significant for our analysis.

No variation is seen with redshift or apparent magnitude. The relationship with SFR shows greater scatter. We find a mean difference of $\log \mathrm{SFR}=0.115$ and variance 0.216 , with estimates from PÉGASE. 2 being larger. This is not surprising, primarily as PÉGASE. 2 estimates the mean SFR averaged over the last 0.5Gyr, while Brinchmann et al. (2004) attempt to determine the instantaneous SFR.

Nevertheless, the PÉGASE.2 and Brinchmann et al. (2004) values agree to within $30 \%$ while the star-forming rates span over two orders of magnitude. No variation is seen as a function of redshift or apparent magnitude. We note that this analysis has primarily considered passive and star-forming galaxies separately, which does not require an accurate measurement of the star-forming rate.

\section{APPENDIX E}

\section{REST-WAVELENGTH COVERAGE}

Throughout this analysis, we have compared our observations at $z<0.25$ to those at higher redshift (Sullivan et al. 2006). While our methodology is identical to that used by Sullivan et al. (2006), both analyses use filter sets that cover the same observed wavelength range, and thus the PÉGASE.2 SED fits are carried out over different rest-wavelength ranges. To test how this difference may affect our conclusions, specifically the comparison to Sullivan et al. (2006), we consider how carrying out our PÉGASE. 2 fits using a reduced number of filters affects the derived stellar mass and SFRs.

The analysis of Sullivan et al. (2006) covers a bluer part of the rest-wavelength spectrum than our analysis. Thus, to produce a combination of filters that closely mimics their work, we use a reduced number of filters, removing the reddest bands from the fitting process. Specifically, we investigate the cases where only the $u g r$ and ugri filters are used.

Figures 12 and 13 show the difference, as a function of apparent magnitude, between the stellar masses and SFRs derived by the PÉGASE. 2 SEDs when various filter combinations are used. As the number of filters is reduced, and thus the number of data points used in the PÉGASE. 2 fits is reduced, the scatter between the stellar mass and SFR distribution is increased. However, in the case where four filters are considered, no significant offset is seen, with a mean difference of $\log M=-0.02$, where galaxies are determined to be slightly less massive when only four filters are used. The SFRs are well recovered, with a mean difference of $\log$ SFR $=-0.024$ and scatter $\sigma=0.141$. There is no evidence that this result is dependent on the magnitude of the galaxy. Altering the number of filters used in the PÉGASE.2 fits allows each galaxy to be classified differently. Of the 85 galaxies that were considered passive when five filters are used, only three $(3.6 \%)$ are classified as star forming, when the $z$ band is omitted.

When only three filters are used to determine the derived parameters, the observed scatter increases as expected for both the stellar mass and SFR distributions. A mean difference of $\log M=-0.12$ with scatter $\sigma=0.23$ is seen. For the SFR distribution, a scatter with mean difference $\log \mathrm{SFR}=-0.12$ and $\sigma=0.23$ is found. Ten galaxies $(11.8 \%)$ that were considered to be passive when all five filters were considered are classified as star forming when only the $u g r$ filters are used in the PÉGASE.2 fits. Four galaxies (1.5\%) that were previously classified as star forming are determined to be passive when only three filters are used. No trend with apparent magnitude is evident in either case.

Since the extra information from the $i$ and $z$ filters does not seem to significantly cause an offset in the PÉGASE.2 fits to higher or lower stellar masses or SFRs, it appears that the restwavelength coverage does not affect our comparisons to Sullivan et al. (2006).

\section{REFERENCES}

Abazajian, K. N., Adelman-McCarthy, J. K., Agüeros, M. A., et al. 2009, ApJS, 182,543

Adelman-McCarthy, J. K., Agüeros, M. A., Allam, S. S., et al. 2006, ApJS, 162, 38

Aldering, G., Antilogus, P., Bailey, S., et al. 2006, ApJ, 650, 510

Baldry, I. K., Balogh, M. L., Bower, R. G., et al. 2006, MNRAS, 373, 469

Baldry, I. K., \& Glazebrook, K. 2003, ApJ, 593, 258

Blanton, M. R., \& Roweis, S. 2007, AJ, 133, 734

Bolzonella, M., Miralles, J.-M., \& Pelló, R. 2000, A\&A, 363, 476

Branch, D., Livio, M., Yungelson, L. R., Boffi, F. R., \& Baron, E. 1995, PASP, 107,1019

Brandt, T. D., Tojeiro, R., Aubourg, É., et al. 2010, AJ, 140, 804

Brinchmann, J., Charlot, S., White, S. D. M., et al. 2004, MNRAS, 351, 1151

Brinchmann, J., \& Ellis, R. S. 2000, ApJ, 536, L77

Calzetti, D., Armus, L., Bohlin, R. C., et al. 2000, ApJ, 533, 682

Cappellaro, E., Evans, R., \& Turatto, M. 1999, A\&A, 351, 459

Cardelli, J. A., Clayton, G. C., \& Mathis, J. S. 1989, ApJ, 345, 245

Chakravarti, I. M., Laha, R. G., \& Roy, J. 1967, Handbook of Methods of Applied Statistics, Vol. I (USE: Wiley)

Cole, S., Norberg, P., Baugh, C. M., et al. 2001, MNRAS, 326, 255

Conley, A., Guy, J., Sullivan, M., et al. 2011, ApJS, 192, 1

D’Agostini, G. 1995, Nucl. Instrum. Methods Phys. Res. A, 362, 487

Dahlen, T., Strolger, L.-G., Riess, A. G., et al. 2004, ApJ, 613, 189

D’Andrea, C. B., Gupta, R. R., Sako, M., et al. 2011, ApJ, 743, 172

Dilday, B., Kessler, R., Frieman, J. A., et al. 2008, ApJ, 682, 262

Dilday, B., Smith, M., Bassett, B., et al. 2010, ApJ, 713, 1026

Donas, J., Deharveng, J. M., Laget, M., Milliard, B., \& Huguenin, D. 1987, A\&A, 180, 12

Felten, J. E. 1976, ApJ, 207, 700

Fioc, M., \& Rocca-Volmerange, B. 1997, A\&A, 326, 950

Frieman, J. A., Bassett, B., Becker, A., et al. 2008, AJ, 135, 338

Fukugita, M., Ichikawa, T., Gunn, J. E., et al. 1996, AJ, 111, 1748

Gallagher, J. S., Garnavich, P. M., Berlind, P., et al. 2005, ApJ, 634, 210

Gal-Yam, A., \& Maoz, D. 2004, MNRAS, 347, 942

Garnavich, P. M., Bonanos, A. Z., Krisciunas, K., et al. 2004, ApJ, 613, 1120

Giavalisco, M., Dickinson, M., Ferguson, H. C., et al. 2004, ApJ, 600, L103

Glazebrook, K., Abraham, R. G., McCarthy, P. J., et al. 2004, Nature, 430, 181

González-Gaitán, S., Perrett, K., Sullivan, M., et al. 2011, ApJ, 727, 107

Grazian, A., Fontana, A., de Santis, C., et al. 2006, A\&A, 449, 951

Greggio, L. 2005, A\&A, 441, 1055

Gunn, J. E., Carr, M., Rockosi, C., et al. 1998, AJ, 116, 3040

Gunn, J. E., Siegmund, W. A., Mannery, E. J., et al. 2006, AJ, 131, 2332

Gupta, R. D., D’Andrea, C. B., Sako, M., et al. 2011, ApJ, 740, 92

Guy, J., Astier, P., Baumont, S., et al. 2007, A\&A, 466, 11

Guy, J., Sullivan, M., Conley, A., et al. 2010, A\&A, 523, A7

Guzman, R., Gallego, J., Koo, D. C., et al. 1997, ApJ, 489, 559

Höflich, P., Gerardy, C., Linder, E., et al. 2003, in Stellar Candles for the Extragalactic Distance Scale, ed. D. Alloin \& W. Gieren (Lecture Notes in Physics, Vol. 635; Berlin: Springer), 203 
Holtzman, J. A., Marriner, J., Kessler, R., et al. 2008, AJ, 136, 2306

Hopkins, A. M., \& Beacom, J. F. 2006, ApJ, 651, 142

Hopkins, A. M., \& Beacom, J. F. 2008, ApJ, 682, 1486

Howell, D. A., Sullivan, M., Conley, A., \& Carlberg, R. 2007, ApJ, 667, L37

Hoyle, F., \& Fowler, W. A. 1960, ApJ, 132, 565

Jha, S., Riess, A. G., \& Kirshner, R. P. 2007, ApJ, 659, 122

Kasen, D., \& Woosley, S. E. 2007, ApJ, 656, 661

Kauffmann, G., Heckman, T. M., White, S. D. M., et al. 2003, MNRAS, 341, 33

Kelly, P. L., Hicken, M., Burke, D. L., Mandel, K. S., \& Kirshner, R. P. 2010, ApJ, 715,743

Kessler, R., Bassett, B., Belov, P., et al. 2010, PASP, 122, 1415

Kessler, R., Becker, A. C., Cinabro, D., et al. 2009a, ApJS, 185, 32

Kessler, R., Bernstein, J. P., Cinabro, D., et al. 2009b, PASP, 121, 1028

King, I. R. 1980, ApJ, 241, 474

Konishi, K., Cinabro, D., Garnavich, P. M., et al. 2011, arXiv:1101.4269

Kroupa, P. 2001, MNRAS, 322, 231

Lampeitl, H., Nichol, R. C., Seo, H.-J., et al. 2010a, MNRAS, 401, 2331

Lampeitl, H., Smith, M., Nichol, R. C., et al. 2010b, ApJ, 722, 566

Le Borgne, D., \& Rocca-Volmerange, B. 2002, A\&A, 386, 446

Le Borgne, D., Rocca-Volmerange, B., Prugniel, P., et al. 2004, A\&A, 425, 881

Leibundgut, B., Kirshner, R. P., Phillips, M. M., et al. 1993, AJ, 105, 301

Li, W., Chornock, R., Leaman, J., et al. 2011a, MNRAS, 412, 1473

Li, W., Filippenko, A. V., Della Valle, M., \& Panagia, N. 2011b, MNRAS, 412, 1508

Maeda, K., Leloudas, G., Taubenberger, S., et al. 2011, MNRAS, 413, 3075

Mannucci, F., Della Valle, M., Panagia, N., et al. 2005, A\&A, 433, 807

Mannucci, F., Della Valle, M., \& Panagia, N. 2006, MNRAS, 370, 773

Maoz, D., Mannucci, F., Li, W., et al. 2011, MNRAS, 412, 1508
Maoz, D., Sharon, K., \& Gal-Yam, A. 2010, ApJ, 722, 1879

Nobili, S., \& Goobar, A. 2008, A\&A, 487, 19

Oemler, A., Jr., \& Tinsley, B. M. 1979, AJ, 84, 985

Oyaizu, H., Lima, M., Cunha, C. E., et al. 2008, ApJ, 674, 768

Phillips, M. M. 1993, ApJ, 413, L105

Prieto, J. L., Garnavich, P. M., Phillips, M. M., et al. 2007, arXiv:0706.4088

Sako, M., Bassett, B., Becker, A., et al. 2008, AJ, 135, 348

Sako, M., Bassett, B., Connolly, B., et al. 2011, ApJ, 738, 162

Salim, S., Charlot, S., Rich, R. M., et al. 2005, ApJ, 619, 39

Sargsyan, L. A., Weedman, D. W., \& Houck, J. R. 2010, ApJ, 715, 986

Scannapieco, E., \& Bildsten, L. 2005, ApJ, 629, L85

Schlegel, D. J., Finkbeiner, D. P., \& Davis, M. 1998, ApJ, 500, 525

Schmidt, M. 1968, ApJ, 151, 393

Sollerman, J., Mörtsell, E., Davis, T. M., et al. 2009, ApJ, 703, 1374

Stoughton, C., Lupton, R. H., Bernardi, M., et al. 2002, AJ, 123, 485

Strateva, I., Ivezić, Ž., Knapp, G. R., et al. 2001, AJ, 122, 1861

Strauss, M. A., Weinberg, D. H., Lupton, R. H., et al. 2002, AJ, 124, 1810

Sullivan, M., Conley, A., Howell, D. A., et al. 2010, MNRAS, 406, 782

Sullivan, M., Guy, J., Conley, A., et al. 2011, ApJ, 737, 102

Sullivan, M., Le Borgne, D., Pritchet, C. J., et al. 2006, ApJ, 648, 868

Suzuki, N., Rubin, D., Lidman, C., et al. 2012, ApJ, 746, 85

Taylor, E. N., Franx, M., van Dokkum, P. G., et al. 2009, ApJ, 694, 1171

Thomas, D., Maraston, C., Schawinski, K., Sarzi, M., \& Silk, J. 2010, MNRAS, 404, 1775

Totani, T., Morokuma, T., Oda, T., Doi, M., \& Yasuda, N. 2008, PASJ, 60, 1327

Wake, D. A., Nichol, R. C., Eisenstein, D. J., et al. 2006, MNRAS, 372, 537

Wang, L., Hoeflich, P., \& Wheeler, J. C. 1997, ApJ, 483, L29

York, D. G., Adelman, J., Anderson, J. E., Jr., et al. 2000, AJ, 120, 1579

Yungelson, L., \& Livio, M. 1998, ApJ, 497, 168

Zheng, C., Romani, R. W., Sako, M., et al. 2008, AJ, 135, 1766 